\title{
Channel Coordination in Logistics Service Supply Chain considering Fairness
}

\author{
Ningning Wang, ${ }^{1}$ Zhi-Ping Fan, ${ }^{1,2}$ and Xiaohuan Wang ${ }^{1}$ \\ ${ }^{1}$ Department of Information Management and Decision Sciences, School of Business Administration, \\ Northeastern University, Shenyang 110169, China \\ ${ }^{2}$ State Key Laboratory of Synthetical Automation for Process Industries, Northeastern University, Shenyang 110819, China
}

Correspondence should be addressed to Xiaohuan Wang; xhwang@mail.neu.edu.cn

Received 11 January 2016; Accepted 6 March 2016

Academic Editor: Sri Sridharan

Copyright (C) 2016 Ningning Wang et al. This is an open access article distributed under the Creative Commons Attribution License, which permits unrestricted use, distribution, and reproduction in any medium, provided the original work is properly cited.

\begin{abstract}
Logistics service supply chain (LSSC) is a new type of service supply chain. This paper investigates the channel coordination issue in a two-echelon LSSC composed of one logistics service integrator (LSI) and one functional logistics service provider (FLSP) under fairness concerns. The models for a reservation price-only contract under disadvantageous inequality and advantageous inequality are established, respectively, in which the procurement cost, the potential shortage cost, and the operation cost are considered under stochastic market demand. Based on this model, the LSI's optimal reservation quantity can be determined. Furthermore, we analyze the impact of fairness concerns and the related costs on channel performance and channel coordination. The results are presented in four aspects: (1) channel coordination of the LSSC can be achieved under certain conditions when the LSI experiences advantageous inequality; (2) the spiteful behavior of the LSI leads to the reduction of the channel profit, and channel coordination cannot be achieved when the LSI suffers from disadvantageous inequality; (3) the LSI's reservation quantity and the channel profit are affected by the LSI's fairness concerns; (4) motivated by the concerns of fairness, the LSI's reservation quantity is related not only to his procurement cost and shortage cost but also to the FLSP's operation cost.
\end{abstract}

\section{Introduction}

Logistics service supply chain (LSSC) refers to a logistics service network structure which is connected from the front-end functional logistics service providers (FLSPs) to the logistics service integrators (LSIs) and to customers (manufacturers or retailers) [1, 2]. It is an important branch of service supply chain focused on the cooperation of logistics service capacities $[3,4]$. In the freight distribution channel, the FLSP and the LSI are urged to work efficiently and collaboratively to maintain their competitiveness [5]. Traditionally, the LSI tends to reserve enough logistics service capacity (e.g., air cargo spaces or container trucks) from the FLSP and then satisfies the market demands of customers. For example, China COSCO logistics company reserves railway transportation service and airline transportation service from TRAINOSE company of Greece and China Southern Airlines (CSA), respectively. Then he provides the comprehensive logistics service to several giants of appliances manufacturers such as
Haier, TCL, and Changhong. In this LSSC, TRAINOSE and CSA play the role of FLSP, while COSCO plays the role of LSI.

In general, the traditional supply chain refers to an integrated manufacturing process including purchasing raw materials, processing raw materials into final products, and distributing the final products to end customers [6, 7], while the LSSC refers to a cooperation process of logistics service capacity, where logistics service is an execution of activity, rather than a tangible asset. Obviously, there are many differences between the traditional supply chain and the LSSC. In a LSSC, the LSI owns the access right of the FLSP's assets (e.g., air cargo spaces or container trucks) rather than the ownership. Thus, the unused logistics service capacity is hard to be resold to the other LSI(s) or FLSP(s) since the negotiation process between the companies (e.g., the LSI and the FLSP) is complicated and the negotiation cost is high; that is, the unused logistics service capacity is costly to be handled as salvage value. Moreover, the 
logistics service has the characteristics such as nonstorage, perishability, and demand uncertainty [8-10]. Hence, the LSI would bear more market risk under the uncertain market demand.

In the LSSC, the conflict and competition widely exist in the cooperation of logistics service capacities since the LSI and the FLSP are independent economic entities [11]. Therefore, channel coordination issue of the LSSC has increasingly caught the attentions in supply chain studies $[4,11]$. The objective of channel coordination is to reduce the negative effects of conflict and demand uncertainty and eventually improve the performance of the supply chain [12-14].

The supply chain coordination issue considering fairness concerns is a new research topic in recent years [15-18]. The existing studies have shown that firms care not only about their own profits but also about whether the profit allocation of the entire channel is fair or not [19-21], that is, fairness concerns [22]. Usually, firms tend to penalize the unfair behaviors even at the cost of paying expense [16, $17,23]$. In a LSSC, the LSI may reduce (or increase) the reservation quantity of logistics service to punish (or reward) the FLSP if he feels unfair. In addition, fairness concerns play an important role in developing and maintaining channel relationship [24], while the sound cooperative relation in a LSSC can reduce the transaction cost and the cooperative risk [25].

Therefore, based on the current studies about supply chain coordination with fairness concerns, whether the profit allocation in the LSSC is fair or not is also a noteworthy problem. Furthermore, given the differences between the traditional supply chain and the LSSC, we would like to focus on several interesting questions: How does the LSI decide the reservation quantity of logistics service under fairness concerns? How do fairness concerns influence the profit allocation in a LSSC? Whether a simple reservation priceonly contract can effectively coordinate the LSI and the FLSP under fairness concerns? If it can, what fairness preference and condition can guarantee the LSSC coordination? How do the related costs (e.g., the LSI's procurement cost and shortage cost and the FLSP's operation cost) impact the LSI's reserving decision towards logistics service capacity?

Two streams of the literature are most closely related to the research issue we are concerned with. The first stream focuses on the channel coordination issue of the LSSC (see [4, 11, 25]). For example, Liu et al. [4] investigate how to determine the fairest revenue-sharing coefficient for the revenue-sharing contract in the LSSC under stochastic demand. By modeling a Stackelberg differential game, Zhao et al. [11] show that the cost sharing contract is an effective coordination mechanism in a LSSC. The second stream investigates the role of fairness concerns in the traditional supply chain (see $[15-18,24,26,27])$. For example, Cui et al. [15] adopt the disutility factor and the equitable payoff factor to depict fairness concerns and investigate how fairness concerns impact channel coordination in a dyadic channel under linear demand. Caliskan-Demirag et al. [26] extend the Cui et al.s [15] model to the situation of nonlinear demand. Similar to the findings of Cui et al. [15] and Caliskan-Demirag et al. [26], Yang et al. [24] find that co-op advertising can coordinate the entire channel in some cases when the retailer has fairness concerns. Du et al. [16] investigate how the fairness preference impacts the channel efficiency by introducing Nash bargaining solution as the fairness reference point. Wu and Niederhoff [18] analyze the impact of fairness concerns for the two-stage random demand newsvendor model.

Our study is different from the above two streams of literature. Comparing to the first literature stream, we consider adopting the fair utility function to describe the LSI's fairness concerns and focus on the impact of the LSI's fairness concerns on the channel performance and channel coordination. Also, we focus on the reservation price-only contract rather than the revenue-sharing contract or the cost sharing contact, since the reservation price-only contract is simple and the incentive contract in the real world frequently takes simpler form than what theory often predicts [2830]. Comparing to the second literature stream, there are two main differences in our study. On the one hand, for the LSSC, the existing studies about the fairness analysis in the traditional supply chain could be unsuitable to some extent since the LSI faces more market risks than the retailer in the traditional supply chain. Thus, we focus on how fairness concerns impact channel performance and channel coordination in a LSSC. On the other hand, most of the existing studies about the fairness analysis in supply chain rarely consider the procurement cost, the potential shortage cost, and the salvage value under stochastic market demand, which are major concerns that could affect the channel members' decisions in reality [31-33]. Thus, to be more close to the reality, we attempt to build the fairness model considering the above-mentioned costs and the operation cost as well.

In this paper, we will start with a benchmark model. Then, based on the benchmark model, the reservation price models considering the LSI's fairness concerns are established; that is, two decentralized models are established under disadvantageous inequality and advantageous inequality, respectively. Through the established models, we derive the closed-form expression of the optimal reservation quantity and investigate the impacts of fairness concerns and the related costs (e.g., the LSI's procurement cost and shortage cost and the FLSP's operation cost) on channel performance and channel coordination.

The major contributions of our study to the research are as follows: we introduce fairness concerns into a two-echelon LSSC and investigate the impacts of fairness concerns on the LSI's reservation quantity, the channel profit, and the channel coordination. We build the fairness model considering the LSI's procurement cost, the potential shortage cost and salvage value, and the FLSP's operation cost under stochastic demand and discuss the impacts of the procurement cost, the shortage cost, and the FLSP's operation cost on the LSI's reservation quantity in detail. Also, we give some valuable management insights for the practices of channel coordination in the LSSC.

The remainder of the paper is organized as follows. In Section 2, we describe the problem and give the 
assumptions and notations used in this paper. In Section 3, we first present the benchmark model, and then we establish the decentralized models under disadvantageous and advantageous inequality. Further, we analyze the impacts of fairness concerns and the related costs on channel performance and channel coordination. In Section 4, several numerical examples are presented to validate the models and the analysis results. Finally, the conclusions of our study and the directions for the future research are presented in Section 5 .

\section{Assumptions and Notations}

In this paper, we consider a one-period two-echelon LSSC composed of one FLSP (she) and one LSI (he). We assume that the market demand of logistics service is stochastic because of demand uncertainty of logistics service. Let $D$ denote the market demand throughout the selling season, $D>0$, and $D$ is drawn from a continuous and differentiable cumulative distribution function $F(x)$ with positive density $f(x), F(0)=0$, and $\mu=E[D]$. Before the selling season, the LSI needs to determine the reservation quantity $q$ of logistics service from the professional FLSP according to his market demand predictions. The FLSP supplies the LSI with the logistics service capacity at the reservation price $w$ per unit. Then the LSI sells comprehensive logistics service to his customers at a unit price $p$. Here, both $p$ and $w$ are exogenous. In this way, the LSI will incur marginal procurement cost $c_{m}$, such as the administrative expenses during the procurement process; meanwhile, the FLSP will incur relevant operation cost $c_{o}$ for offering logistics service capacity. Furthermore, the LSI will pay a goodwill penalty $\operatorname{cost} c_{s}$ (shortage cost) to customers when he lacks logistics service capacity. When the LSI has surplus capacity, the LSI earns the salvage value $v_{e}$ per unit of unused logistics service capacity at the end of season. We assume that $v_{e}$ can be deemed as zero since the unused logistics service cannot be stored or is costly to be resold to the other LSI(s) or FLSP(s). Thus, the LSI would bear all the potential risks caused by capacity shortage or surplus in the situation of uncertain market. In view of the actual situation, without loss of generality, we assume that $c_{s}>w>c_{o}>c_{m}$.

In the problem that we are concerned with, we assume that both the FLSP and the LSI are risk-neutral and information-symmetrical. The LSI has his own fairness benchmark; that is, the equitable outcome for the LSI is $\gamma$ times the FLSP's outcome. That is to say, the LSI will perceive disadvantageous inequality if his profit is less than $\gamma$ times that of the FLSP's outcome; in the meantime, he will perceive advantageous inequality if his profit is greater than $\gamma$ times that of the FLSP's outcome. Specifically, we follow the same route as Cui et al. [15]; the LSI's expected utility $U_{I}$ can be expressed by

$$
\begin{aligned}
U_{I}= & \Pi_{I}-\alpha \max \left\{\gamma \Pi_{F}-\Pi_{I}, 0\right\} \\
& -\beta \max \left\{\Pi_{I}-\gamma \Pi_{F}, 0\right\},
\end{aligned}
$$

where $\Pi_{I}$ and $\Pi_{F}$ denote the expected profits of the LSI and the FLSP, respectively. $\alpha, \beta$, and $\gamma$ are three coefficients depicting fairness concerns. $\alpha$ denotes the measurement of the LSI's disutility or inequity-averse degree if his profit is less than his equitable outcome [18], $\alpha>0 . \beta$ denotes the measurement of the LSI's disutility or inequity-averse degree if his profit is greater than his equitable outcome $[18,34], \beta \leq \alpha$ and $0<\beta<1 . \gamma$ denotes the degree of LSI's perceived relative advantage against the FLSP and depends on the factors such as the outside options and so on $[15,27], \gamma>0$. Specially, the larger the LSI's perceived relative advantage against the FLSP is, the bigger the $\gamma$ will be.

To facilitate the following analysis, we summarize the key notations as shown in the following part.

\section{Variable Definitions}

$D$ : market demand of logistics service,

$f(x)$ : probability density function of market demand $D$,

$F(x)$ : cumulative distribution function of market demand $D$,

$c_{m}$ : LSI's marginal procurement of logistics service capacity,

$c_{o}$ : FLSP's unit operation cost of logistics service capacity,

$c_{s}$ : the unit shortage cost of insufficient logistics service capacity,

$v_{e}$ : the unit salvage value of unused logistics service capacity,

$w$ : reservation price per unit of logistics service capacity,

$p$ : selling price per unit of logistics service capacity,

$q$ : LSI's reservation quantity of logistics service,

$\alpha, \beta$, and $\gamma$ : coefficients in the utility function (1),

$\Pi_{I}$ : expected profit of LSI,

$\Pi_{F}$ : expected profit of FLSP,

$\Pi_{L}$ : expected profit of entire LSSC.

\section{Model Formulation}

Given that the market demand of logistics service is stochastic, the LSI's expected sales $s(q)$ are given by $s(q)=q-$ $\int_{0}^{q} F(x) d x$; the LSI's expected lost-sales $g(q)$ are given by $g(q)=\mu-q+\int_{0}^{q} F(x) d x$ if $q<D$ (the LSI's reserved quantity of logistics service is less than the market demand), and the LSI's expected unused capacity $h(q)$ is given by $h(q)=$ $\int_{0}^{q} F(x) d x$ if $q>D$. In this section, the centralized model and the decentralized model are constructed, which serve as the benchmark to evaluate channel coordination in the LSSC under fairness concerns. Further, the channel coordination models are established when the LSI experiences disadvantageous and advantageous inequality, respectively. On the 
basis of these models, some analyses are given correspondingly.

3.1. Benchmark Model. In the centralized setting, the LSI and the FLSP make decision together, and they hope to maximize the entire channel profit. Thus, the profit of the entire LSSC can be formulated as

$$
\begin{aligned}
\pi_{L}= & p \min (q, D)-\left(c_{m}+c_{o}\right) q-c_{s}(D-q)^{+} \\
& +v_{e}(q-D)^{+} .
\end{aligned}
$$

Since the unused logistics service capacity cannot be stored or is costly to be resold to the other companies, the salvage value of the unused logistics service capacity can be deemed as zero; that is, $v_{e}=0$. Thus, the expected profit of the entire LSSC can be expressed as

$$
\begin{aligned}
\Pi_{L}= & p q-p \int_{0}^{q} F(x) d x-\left(c_{m}+c_{o}\right) q-\mu c_{s}+c_{s} q \\
& -c_{s} \int_{0}^{q} F(x) d x .
\end{aligned}
$$

Since $\partial^{2} \Pi_{L} / \partial q^{2}<0, \Pi_{L}$ is concave with respect to $q$. Therefore, the optimal reservation quantity of the LSSC can be obtained; that is,

$$
q_{\mathrm{cen}}^{*}=F^{-1}\left(1-\frac{c_{m}+c_{o}}{p+c_{s}}\right) \text {. }
$$

In the traditional decentralized setting, all participants maximize their individual profits without considering any fairness issues. The sequence of events in this game is as follows: the FLSP offers the LSI a reservation price-only contract, and then the LSI determines whether the contract is accepted or not. If the LSI accepts the contract, he will seek the optimal reservation quantity of logistics service to maximize his own profit; if the LSI rejects the contract, the game ends and each participant will earn a default payoff. Thus, the LSI's profit can be given by

$$
\begin{aligned}
\pi_{I}= & p \min (q, D)-\left(w+c_{m}\right) q-c_{s}(D-q)^{+} \\
& +v_{e}(q-D)^{+} .
\end{aligned}
$$

Taking $v_{e}=0$ into account, the LSI's expected profit can be formulated as

$$
\begin{aligned}
\Pi_{I}= & p q-p \int_{0}^{q} F(x) d x-\left(w+c_{m}\right) q-\mu c_{s}+c_{s} q \\
& -c_{s} \int_{0}^{q} F(x) d x .
\end{aligned}
$$

The FLSP's expected profit can be formulated as

$$
\Pi_{F}=w q-c_{o} q
$$

Since $\partial^{2} \Pi_{I} / \partial q^{2}<0, \Pi_{I}$ is concave with respect to $q$. Therefore, the optimal reservation quantity of the LSI can be obtained; that is,

$$
q_{\mathrm{dec}}^{*}=F^{-1}\left(1-\frac{w+c_{m}}{p+c_{s}}\right) .
$$

Given that $w>c_{o}$, it can be seen from (4) and (8) that $q_{\text {cen }}^{*}>q_{\mathrm{dec}}^{*}$. Thus, the reservation quantity in the decentralized setting is not equal to the one in the centralized setting due to the double marginalization problem. In other words, the double marginalization problem cannot be eliminated unless the FLSP's profit is zero (i.e., $w=c_{o}$ ). Obviously, the LSSC cannot be coordinated by the reservation priceonly contract when the LSI's procurement cost, the potential shortage cost and salvage value, and the FLSP's operation cost are considered. Also, through the comparison and analysis, we find that the optimal reservation quantity of the LSI is smaller than that of the retailer from a classical newsvendor problem when the LSI faces a greater market risk [12]. In other words, the optimal reservation quantity of the LSI in decentralized setting even derives from that in the centralized setting.

3.2. Model with the LSI's Fairness Concerns. We restrict our attention to a scenario that the LSI is fair-minded in the decentralized LSSC. Since the FLSP's objective is to maximize his profit, her utility is equivalent to her profit. The sequence of events of the game is as follows: the FLSP first provides a reservation price-only contract to the LSI; then, by observing the stochastic demand, the LSI decides whether the contract is accepted or not. If he accepts, he must make the decision about the reservation quantity of logistics service to maximize his utility; otherwise, the game ends. To describe the LSI's expected utilities under disadvantageous and advantageous inequality, respectively, (1) can be expressed in the form of a piecewise function; that is,

$$
U_{I}= \begin{cases}(1+\alpha) \Pi_{I}-\alpha \gamma \Pi_{F}, & \text { for } \Pi_{I} \leq \gamma \Pi_{F} \\ (1-\beta) \Pi_{I}+\beta \gamma \Pi_{F}, & \text { for } \Pi_{I} \geq \gamma \Pi_{F}\end{cases}
$$

From (9), if the LSI's profit is smaller than his equitable outcome, that is, $\Pi_{I} \leq \gamma \Pi_{F}$, then he suffers from disadvantageous inequality and behaves spitefully; if the LSI's profit is greater than his equitable outcome, that is, $\Pi_{I} \geq \gamma \Pi_{F}$, he experiences advantageous inequality and behaves generously.

3.2.1. Disadvantageous Inequality. If the LSI accepts the FLSP's reservation price-only contract and his payoff is smaller than $\gamma$ times that of the FLSP's outcome, then the LSI will determine a desirable reservation quantity to maximize his utility. Thus, according to (6), (7), and (9), the optimization problem faced by the LSI under disadvantageous inequality can be defined as 


$$
\begin{aligned}
\max _{q \geq 0} & U_{\mathrm{dis}}^{I}=(1+\alpha) \Pi_{I}-\alpha \gamma \Pi_{F} \\
& =(1+\alpha)\left[p q-p \int_{0}^{q} F(x) d x-\left(w+c_{m}\right) q-\mu c_{s}+c_{s} q-c_{s} \int_{0}^{q} F(x) d x\right]-\alpha \gamma\left(w-c_{o}\right) q .
\end{aligned}
$$

By (10), we can get the following propositions.

Proposition 1. If the LSI suffers from disadvantageous inequality under the reservation price-only contract, then the optimal reservation quantity, $q_{d i s}^{*}$, is

$$
q_{d i s}^{*}=F^{-1}\left(1-\frac{(1+\alpha)\left(w+c_{m}\right)+\alpha \gamma\left(w-c_{o}\right)}{(1+\alpha)\left(p+c_{s}\right)}\right) .
$$

The proof of Proposition 1 is provided in Appendix. Proposition 1 indicates that the LSI's expected utility, $U_{\text {dis }}^{I}$, is strictly concave with respect to $q$. That is to say, when he accepts the reservation price-only contract and suffers from disadvantageous inequality, his optimal reservation quantity is $q_{\mathrm{dis}}^{*}$.

Proposition 2. The LSI's optimal reservation quantity, $q_{d i s}^{*}$, is affected by coefficients $\alpha$ and $\gamma$. For any $\alpha>0$ and $\gamma>0$, one has the following: (a) $q_{d i s}^{*}$ is decreasing in $\alpha$; (b) $q_{d i s}^{*}$ is decreasing in $\gamma$; and (c) $q_{d i s}^{*}<q_{d e c}^{*}<q_{c e n}^{*}$.

The proof of Proposition 2 is provided in Appendix. The meaning of Proposition 2 is explained as follows. (a) The LSI's actual decision is affected by his inequity-averse degree if he suffers from disadvantageous inequality. That is, if the LSI behaves more spitefully (the averse degree towards disadvantageous inequality is high), his reservation quantity will further deviate from $q_{\text {cen }}^{*}$ to punish the FLSP. (b) The LSI's reservation quantity decreases with the increase of $\gamma$ when a neutral FLSP sells some kinds of logistics services to a spiteful LSI. In other words, the higher the spiteful LSI's perceived relative advantage against the FLSP is, the less the quantity of logistics service he will book. (c) If the LSI suffers from disadvantageous inequality, his spiteful effect will discourage him from making greater efforts on reservation, and his reservation quantity is smaller than the centralized reservation quantity.

Proposition 3. The LSI's optimal reservation quantity, $q_{d i s}^{*}$, is related to his procurement cost $c_{m}$ and shortage cost $c_{s}$ and the FLSP's operation cost $c_{o}$; that is, (a) $q_{d i s}^{*}$ is decreasing in $c_{m}$; (b) $q_{d i s}^{*}$ is increasing in $c_{s}$; and (c) $q_{d i s}^{*}$ is increasing in $c_{o}$.

The proof of Proposition 3 is provided in Appendix. Proposition 3 indicates that when the LSI suffers from disadvantageous inequality, his optimal reservation quantity is related not only to his procurement cost and shortage cost but also to the FLSP's operation cost. Specifically, with the increase of the procurement cost, the LSI will decrease his reservation quantity to avoid paying more procurement cost. With the increase of the shortage cost, the LSI will increase his reservation quantity to avoid capacity shortage. With the increase of the FLSP's operation cost, the LSI will increase his reservation quantity to seek a more fair trade. It can be seen from (8) that the fair-neutral LSI's reservation quantity is only affected by his procurement cost and shortage cost, while the spiteful LSI's reservation quantity is also affected by the FLSP's operation cost. This just reflects the nature of fairness concerns; that is, the LSI cares not only about his own profit but also about the FLSP's profit. Combining with Proposition 2, we observe that the LSI's spiteful effect is restrained by the increase of the FLSP's operation cost.

Proposition 4. If the LSI suffers from disadvantageous inequality, one has the following: (a) $\Pi_{I}^{\text {dis }}, \Pi_{F}^{\text {dis }}$, and $\Pi_{L}^{\text {dis }}$ are all decreasing in $\alpha ;(b) \Pi_{I}^{d i s}, \Pi_{F}^{d i s}$, and $\Pi_{L}^{\text {dis }}$ are all decreasing in $\gamma$.

The proof of Proposition 4 is provided in Appendix. The meaning of Proposition 4 is as follows: (a) profits of the LSI, the FLSP, and the entire LSSC all decrease as the LSI's inequity-averse degree increases; that is, the spiteful effect always leads to the reduction of the LSSC's performance. (b) The higher the LSI's perceived relative advantage against the FLSP is, the less the profits of the participants and the entire LSSC will be obtained. This is common in reality. For example, if a company feels unfairly treated in the cooperation with his partner, he will behave spitefully to his partner. The bigger his contribution to the channel is, the tougher his sanction to his partner will be. That is, the company will decrease his reservation quantity even if his own interest will be hurt, and the partner will be dissatisfied with the collaboration. Sometimes, the partner will counterattack the company's punishment. This will lead to further deterioration of the partnership and reduction of the LSSC's performance.

Proposition 5. If the LSI suffers from disadvantageous inequality, then one has the following: (a) $\Pi_{I}^{\text {dis }}<\Pi_{I}^{\text {dec }}, \Pi_{F}^{d i s}<$ $\Pi_{F}^{d e c}$, and $\Pi_{L}^{\text {dis }}<\Pi_{L}^{\text {dec }}$; (b) channel coordination of the LSSC cannot be achieved.

The proof of Proposition 5 is provided in Appendix. Proposition 5 shows that the optimal profit of the LSSC cannot be achieved through a simple reservation price-only contract if the LSI suffers from disadvantageous inequality, and the coordination effect is affected by the LSI's fairness concerns in the decentralized setting. That is, the double marginalization problem becomes worse if the LSI suffers from disadvantageous inequality. 
3.2.2. Advantageous Inequality. When the LSI accepts the FLSP's reservation price-only contract and his payoff is larger than $\gamma$ times that of the FLSP's outcome, the LSI will reserve enough logistics service capacity to maximize his utility. According to (6), (7), and (9), the optimization problem faced by the LSI under advantageous inequality can be defined as

$$
\begin{aligned}
\max _{q \geq 0} & U_{\mathrm{adv}}^{I}=(1-\beta) \Pi_{I}+\beta \gamma \Pi_{F} \\
& =(1-\beta)\left[p q-p \int_{0}^{q} F(x) d x-\left(w+c_{m}\right) q-\mu c_{s}+c_{s} q-c_{s} \int_{0}^{q} F(x) d x\right]+\beta \gamma\left(w-c_{o}\right) q .
\end{aligned}
$$

By (12), we can get the following propositions.

Proposition 6. If the LSI experiences advantageous inequality under the reservation price-only contract, the optimal reservation quantity, $q_{a d v}^{*}$, is

$$
q_{a d v}^{*}=F^{-1}\left(1-\frac{(1-\beta)\left(w+c_{m}\right)-\beta \gamma\left(w-c_{o}\right)}{(1-\beta)\left(p+c_{s}\right)}\right) .
$$

The proof of Proposition 6 is provided in Appendix. Proposition 6 indicates that the LSI's expected utility, $U_{\mathrm{adv}}^{I}$, is strictly concave with respect to $q$. That is to say, when the LSI accepts the reservation price-only contract and experiences advantageous inequality, his optimal reservation quantity is $q_{\mathrm{adv}}^{*}$.

Proposition 7. The optimal reservation quantity, $q_{a d v}^{*}$, is affected by the coefficient $\beta$. For any $\gamma>0$, one has the following: (a) $q_{a d v}^{*}$ is increasing in $\beta$; (b) $q_{d e c}^{*}<q_{a d v}^{*}<q_{c e n}^{*}$ if $0<\beta<1 /(1+\gamma)$; (c) $q_{d e c}^{*}<q_{a d v}^{*}=q_{c e n}^{*}$ if $\beta=1 /(1+\gamma)$; and $(d) q_{d e c}^{*}<q_{c e n}^{*}<q_{a d v}^{*}$ if $1 /(1+\gamma)<\beta<1$.

The proof of Proposition 7 is provided in Appendix. Proposition 7 indicates that if the LSI experiences advantageous inequality, his reservation quantity, $q_{\mathrm{adv}}^{*}$, will go up as his inequity-averse degree increases. In other words, if the LSI is insufficiently averse to advantageous inequality, then $q_{\mathrm{adv}}^{*}<$ $q_{\text {cen }}^{*}$; if the LSI is sufficiently averse to advantageous inequality (i.e., $\beta=1 /(1+\gamma)$ ), then $q_{\mathrm{adv}}^{*}=q_{\mathrm{cen}}^{*}$; if the LSI is excessively averse to advantageous inequality, then $q_{\mathrm{adv}}^{*}>q_{\mathrm{cen}}^{*}$. Besides, in the decentralized setting, the reservation quantity of the generous LSI is bigger than that of the neutral LSI.

Proposition 8. The optimal reservation quantity, $q_{a d v}^{*}$, is affected by the coefficient $\gamma$. For any $0<\beta<1$, one has the following: (a) $q_{a d v}^{*}$ is increasing in $\gamma$; (b) $q_{d e c}^{*}<q_{a d v}^{*}<q_{c e n}^{*}$ if $0<\gamma<(1-\beta) / \beta$; (c) $q_{d e c}^{*}<q_{a d v}^{*}=q_{\text {cen }}^{*}$ if $\gamma=(1-\beta) / \beta$; and (d) $q_{d e c}^{*}<q_{c e n}^{*}<q_{a d v}^{*}$ if $\gamma>(1-\beta) / \beta$.

The proof of Proposition 8 is provided in Appendix. Proposition 8 indicates that the LSI's reservation quantity, $q_{\mathrm{adv}}^{*}$, is increasing in $\gamma$ when a neutral FLSP sells some kinds of logistics service to a generous LSI. In other words, the greater the generous LSI's perceived relative advantage against the FLSP is, the larger the quantity of logistics service he will reserve. When the LSI's perceived relative advantage against the FLSP is satisfied with $\gamma=(1-\beta) / \beta$, his optimal booking strategy is the same as the one in the centralized setting.
Proposition 9. The LSI's optimal reservation quantity, $q_{a d v}^{*}$, is related to his procurement cost $c_{m}$ and shortage cost $c_{s}$ and the FLSP's operation cost $c_{o}$; that is, (a) $q_{a d v}^{*}$ is decreasing in $c_{m}$; (b) $q_{a d v}^{*}$ is increasing in $c_{s}$; and (c) $q_{a d v}^{*}$ is decreasing in $c_{o}$.

The proof of Proposition 9 is provided in Appendix. Proposition 9 shows that a generous LSI's reservation quantity is affected not only by his procurement cost and shortage cost but also by the FLSP's operation cost. Specifically, the LSI's reservation quantity will decrease as his procurement cost increases. With the increase of the shortage cost, the LSI will increase his reservation quantity to avoid capacity shortage. With the increase of FLSP's operation cost, the LSI will decrease his reservation quantity so as to seek a more fair trade. Combining with Proposition 7, we observe that the LSI's generous effect will be restrained by the increase of the operation cost.

Proposition 10. In the decentralized setting, when the LSI experiences advantageous inequality, one has the following: (a) $\Pi_{I}^{a d v}$ is decreasing in $\beta, \Pi_{F}^{a d v}$ is increasing in $\beta$, and $\Pi_{L}^{a d v}$ is increasing (decreasing) in $\beta$ if $\beta<1 /(1+\gamma)(\beta>1 /(1+\gamma))$; (b) $\Pi_{I}^{a d v}$ is decreasing in $\gamma, \Pi_{F}^{a d v}$ is increasing in $\gamma$, and $\Pi_{L}^{a d v}$ is increasing (decreasing) in $\gamma$ if $\gamma<(1-\beta) / \beta(\gamma<(1-\beta) / \beta)$.

The proof of Proposition 10 is provided in Appendix. The meaning of Proposition 10 is as follows. (a) Profit allocation of the LSSC is affected by the LSI's averse degree towards advantageous inequality $(\beta)$. With the increase of $\beta$, the LSI will shift part of his monetary payoff to the FLSP, and the entire channel profit first experiences an increase and then follows a decline; that is, the LSI's generous effect has an important role to improve (reduce) the LSSC's performance when $\beta<\beta_{1}\left(\beta>\beta_{1}\right)$. (b) Profit allocation of the LSSC is also affected by the coefficient $\gamma$, and the impact of $\gamma$ is the same as $\beta$. That is, the bigger the LSI's perceived relative advantage against the FLSP is, the more he will tend to shift his profit to the FLSP.

Proposition 11. In the situation that the LSI experiences advantageous inequality, channel coordination in the LSSC can be achieved if $\beta=1 /(1+\gamma)$.

The proof of Proposition 11 is provided in Appendix. Proposition 11 shows that the reservation price-only contract cannot coordinate the entire channel in the LSSC when the LSI does not have fairness concerns or the LSI experiences 
TABLE 1: The influences of coefficients $\alpha, \beta$, and $\gamma$ on the reservation quantities and the channel profits under disadvantageous and advantageous inequality.

\begin{tabular}{lcccccccc}
\hline \multirow{3}{*}{ Coefficients } & \multicolumn{1}{c}{ Disadvantageous inequality } & \multicolumn{3}{c}{ Advantageous inequality } \\
& $q_{\text {dis }}^{*}$ & $\Pi_{I}^{\text {dis }}$ & $\Pi_{F}^{\text {dis }}$ & $\Pi_{L}^{\text {dis }}$ & $q_{\text {adv }}^{*}$ & $\Pi_{I}^{\text {adv }}$ & $\Pi_{F}^{\text {adv }}$ & $\Pi_{L}^{\text {adv }}$ \\
\hline$\alpha$ & $\searrow$ & $\searrow$ & $\nearrow$ & $\searrow$ & - & - & - & - \\
$\gamma$ & $\searrow$ & $\searrow$ & $\nearrow$ & $\searrow$ & $\nearrow$ & $\searrow$ & $\nearrow$ & $\nearrow \searrow$ \\
$\beta$ & - & - & - & - & $\nearrow$ & $\searrow$ & $\nearrow$ & $\nearrow \searrow$ \\
\hline
\end{tabular}

Note: $\searrow$ and $\nearrow$ denote that the result is decreasing and increasing in $\alpha, \beta$, or $\gamma$, respectively, and $\nearrow \backslash$ denotes that the result first goes up and then goes down with the increase of $\alpha, \beta$, or $\gamma$. - denotes that $\alpha, \beta$, or $\gamma$ has no effect on the result.

disadvantageous inequality, but the contract can coordinate the entire channel under the certain condition $(\beta=$ $1 /(1+\gamma))$ when the LSI experiences advantageous inequality.

Next we discuss the changes of profit and utility of the channel members to achieve the above-mentioned channel coordination. If $\beta=1 /(1+\gamma)$, it can be seen from Propositions 9 and 11 that the profit of a generous LSI in the coordinated scenario is smaller than that of a neutral LSI in the decentralized setting (i.e., $\Pi_{I}^{\mathrm{adv}}=\Pi_{I}^{\mathrm{cen}}<\Pi_{I}^{\mathrm{dec}}$ ), and the profits of the FLSP and the entire LSSC are greater than the ones in the traditional decentralized setting (i.e., $\Pi_{F}^{\mathrm{adv}}=\Pi_{F}^{\mathrm{cen}}>\Pi_{F}^{\mathrm{dec}}$ and $\left.\Pi_{L}^{\mathrm{adv}}=\Pi_{L}^{\mathrm{cen}}>\Pi_{L}^{\mathrm{dec}}\right)$. Thus, if the LSI experiences advantageous inequality, the reservation price-only contract is beneficial to both the FLSP and the entire LSSC, but unfavorable to the LSI. Motivated by the concerns of fairness, the LSI will change his objective; that is, profit maximization objective will be changed into utility maximization objective. When the LSI experiences advantageous inequality, his reservation quantity should be $q_{\mathrm{adv}}^{*}$ so as to maximize his utility. Specially, if $\beta=1 /(1+\gamma)$, the generous LSI can not only maximize his utility but also maximize the profit and the total utility of the entire channel; that is, the channel coordination can be achieved. Here, the total utility is the sum of the LSI's utility and the FLSP's profit (utility). Although the LSI's profit in the coordinated situation is smaller than the one in the traditional decentralized setting, the LSI feels more fair; that is, the LSI tends to increase his reservation quantity to avoid feeling more advantageous inequality.

Table 1 summarizes the impacts of the coefficients $\alpha, \beta$, and $\gamma$ on the LSI's reservation quantity and the profits of the channel members in the LSSC under disadvantageous inequality and advantageous inequality. It can be seen from Table 1 that the impacts of $\alpha$ and $\gamma$ ( $\beta$ and $\gamma$ ) on the LSI's decision and the performance of the LSSC are the same if the LSI experiences disadvantageous inequality (advantageous inequality).

\section{Numerical Examples}

In this section, we give several numerical examples under both disadvantageous and advantageous inequality to illustrate the above theoretical results. For the purpose of our numerical demonstration, we consider that the logistics demand in the market follows the normal distribution [18, 35], $D \sim N\left(1000,100^{2}\right)$.

\subsection{Relevant Numerical Examples under Disadvantageous} Inequality. In this subsection, we give two numerical examples under disadvantageous inequality. One is to illustrate the changes of the reservation quantities and the entire channel profits with respect to $\alpha$ and $\gamma$. The other is to explore the impacts of the procurement cost, the shortage cost, and the operation cost on the reservation quantities and the channel members' profits.

Example 1. In this example, to satisfy $c_{s}>w>c_{o}>c_{m}$ and ensure that the profit of the LSI is lower than his equitable profit, we set the parameters as follows: $c_{m}=4, c_{o}=10, w=$ $18, c_{s}=22$, and $p=30$.

Figure 1 presents the results about the impacts of $\alpha$ and $\gamma$ on the reservation quantities and the entire channel profits under disadvantageous inequality. Figure 1(a) shows that the reservation quantity of the spiteful LSI decreases as $\alpha$ or $\gamma$ increases. In the decentralized setting, the reservation quantity of the spiteful LSI is not greater than that of the fair-neutral LSI. In addition, the reservation quantity of both the spiteful and the fair-neutral LSI in the decentralized setting is less than the centralized reservation quantity. These results are in accordance with Proposition 2. Figure 1(b) shows that the entire channel profit is decreasing in $\alpha$ or $\gamma$. In the decentralized setting, the entire channel profit of the disadvantageous inequality scenario is less than that of the fair-neutral scenario, and both of them are less than those of the centralized setting. The results show that channel coordination of the LSSC cannot be achieved when the LSI suffers from disadvantageous inequality. These results are in accordance with Propositions 4 and 5.

Example 2. In this example, we set three cases to analyze the impacts of $c_{m}, c_{s}$, and $c_{o}$ on the reservation quantities and the channel members' profits under disadvantageous inequality. To satisfy $c_{s}>w>c_{o}>c_{m}$ and ensure that the profit of the LSI is lower than his equitable profit, the parameters for the three cases are set as follows.

Case 1. Consider $c_{m} \in[2,9], \alpha=1, \gamma=1, c_{o}=10, w=18$, $c_{s}=22$, and $p=30$.

Case 2. Consider $c_{s} \in[18,25], \alpha=1, \gamma=1, c_{m}=4, c_{o}=10$, $w=18$, and $p=30$.

Case 3. Consider $c_{o} \in[5,12], \alpha=1, \gamma=1, c_{m}=4, w=18$, $c_{s}=22$, and $p=30$.

For Case 1, the results are presented in Figure 2. In Figure 2(a), we can observe that the reservation quantity of the spiteful LSI is decreasing in $c_{f}$, and this result is in accordance with Proposition 3. Figure 2(b) shows that when the LSI experiences disadvantageous inequality, the profit of the LSI and the FLSP will decrease with the increase of the LSI's procurement cost. 


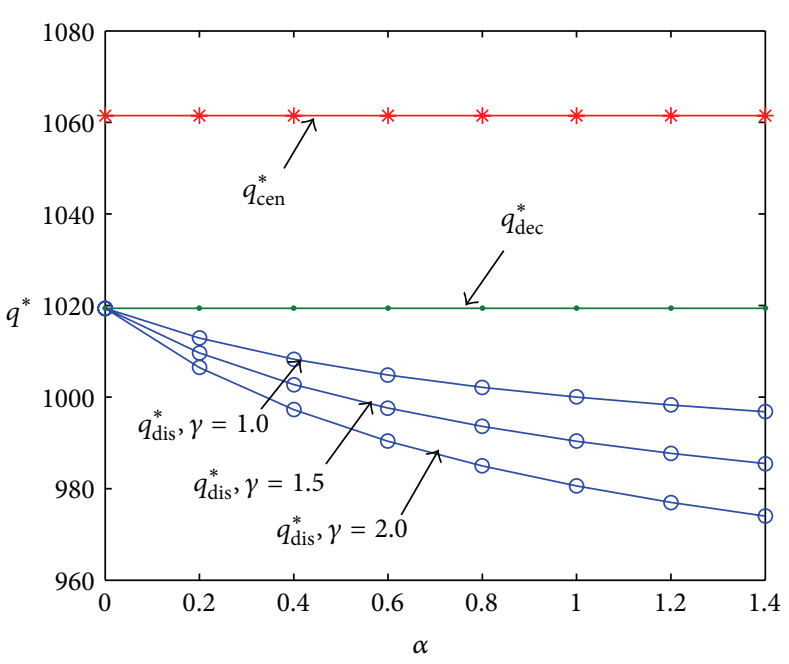

(a) The reservation quantities versus $(\alpha, \gamma)$

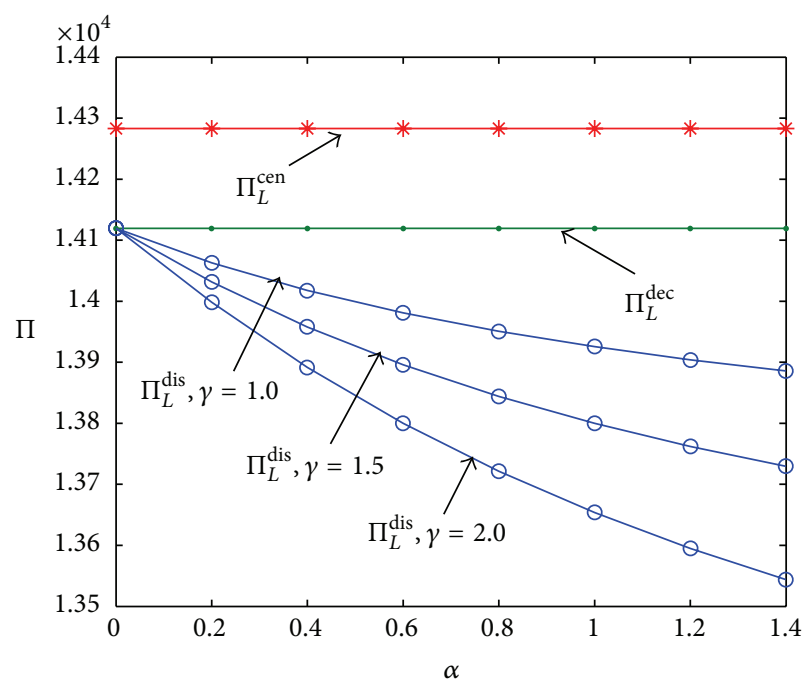

(b) The entire channel profits versus $(\alpha, \gamma)$

FIGURE 1: Impacts of $\alpha$ and $\gamma$ on the reservation quantities and the entire channel profits under disadvantageous inequality.

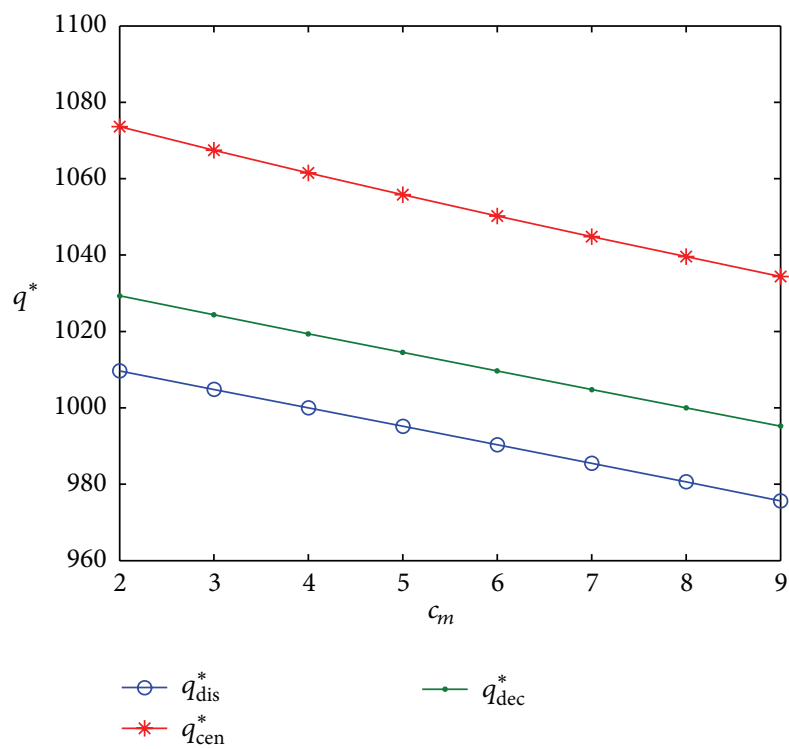

(a) The reservation quantities versus $c_{m}$

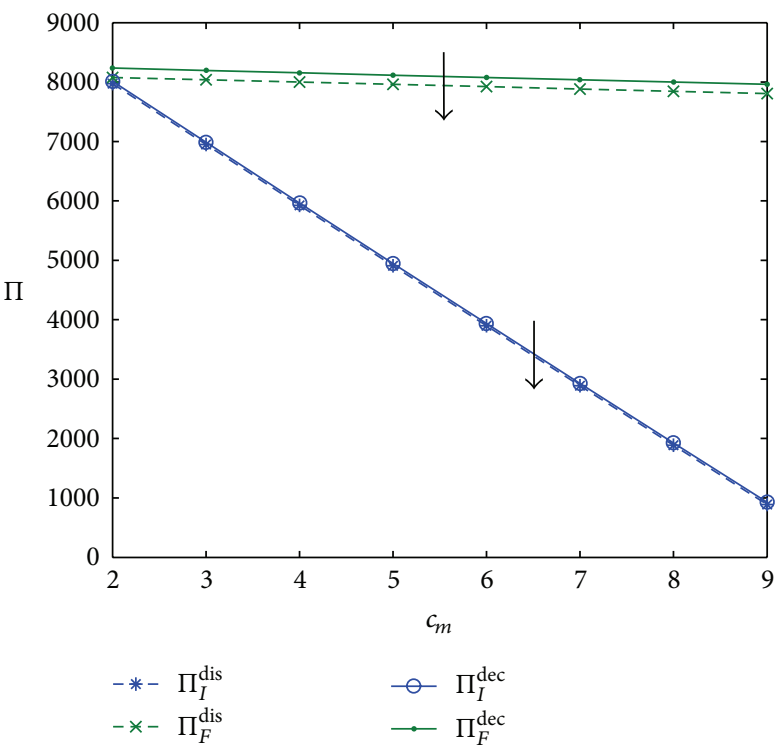

(b) The channel members' profits versus $c_{m}$

FIGURE 2: Impacts of $c_{m}$ on the reservation quantities and the channel members' profits under disadvantageous inequality.

For Case 2, the results are shown in Figure 3. Figure 3(a) shows that the reservation quantity of the spiteful LSI will increase as the shortage cost increases, and this result is in accordance with Proposition 3. Figure 3(b) shows that when the LSI experiences disadvantageous inequality, his profit will decrease as the shortage cost increases, while the FLSP's profit will increase.

For Case 3, the results are presented in Figure 4. Figure 4(a) demonstrates that the reservation quantity of the spiteful LSI is affected by the FLSP's operation cost, and it will increase as the operation cost increases. The results are in accordance with Proposition 3. Figure 4(b) shows that when the LSI suffers from disadvantageous inequality, his profit will increase as the operation cost increases, while the FLSP's profit will decrease.

It can be seen from Figures 1(a), 2(a), and 3(a) that the reservation quantity of the spiteful LSI is always smaller than that of the fair-neutral LSI in the decentralized setting, and the reservation quantities of the spiteful LSI and the fair-neutral LSI are both smaller than the centralized reservation quantity. Moreover, from Figures 1(b), 2(b), and 3(b), we can observe that the profit of both the LSI and the FLSP under fair-neutral scenario is smaller than that in the disadvantageous inequality scenario. This reflects that the LSI tends to punish the FLSP so as to 


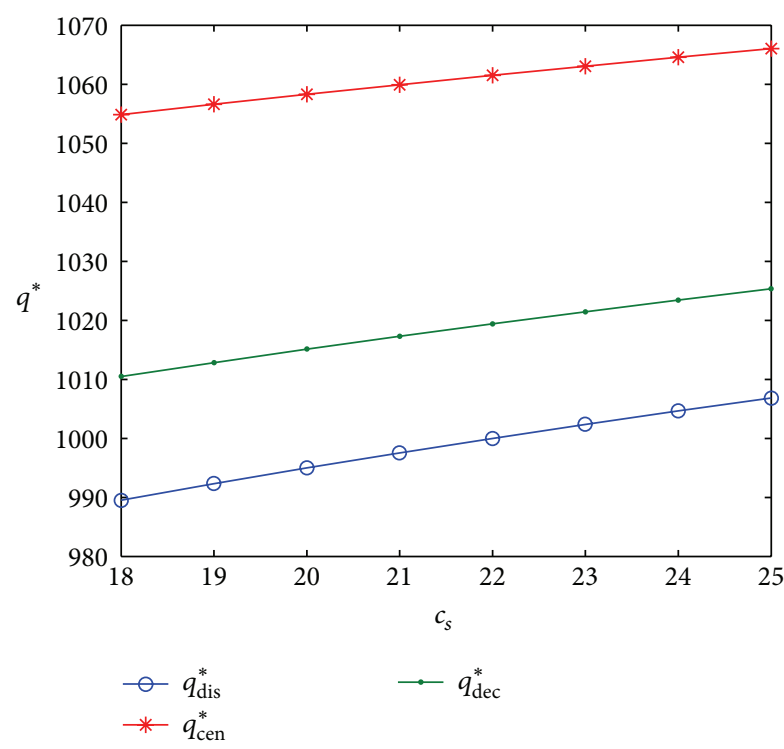

(a) The reservation quantities versus $c_{s}$

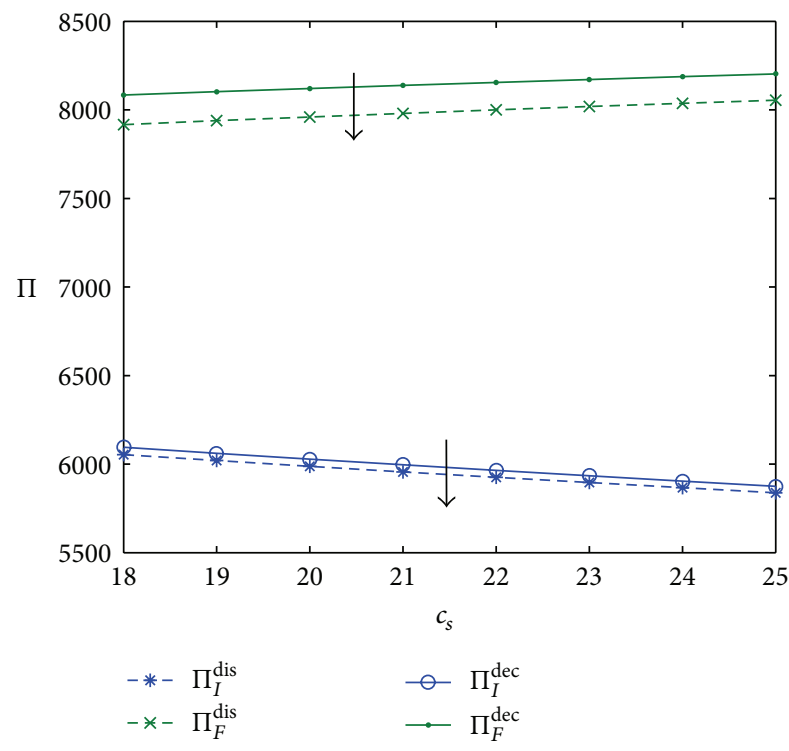

(b) The channel members' profits versus $c_{s}$

FIGURE 3: Impacts of $c_{s}$ on the reservation quantities and the channel members' profits under disadvantageous inequality.

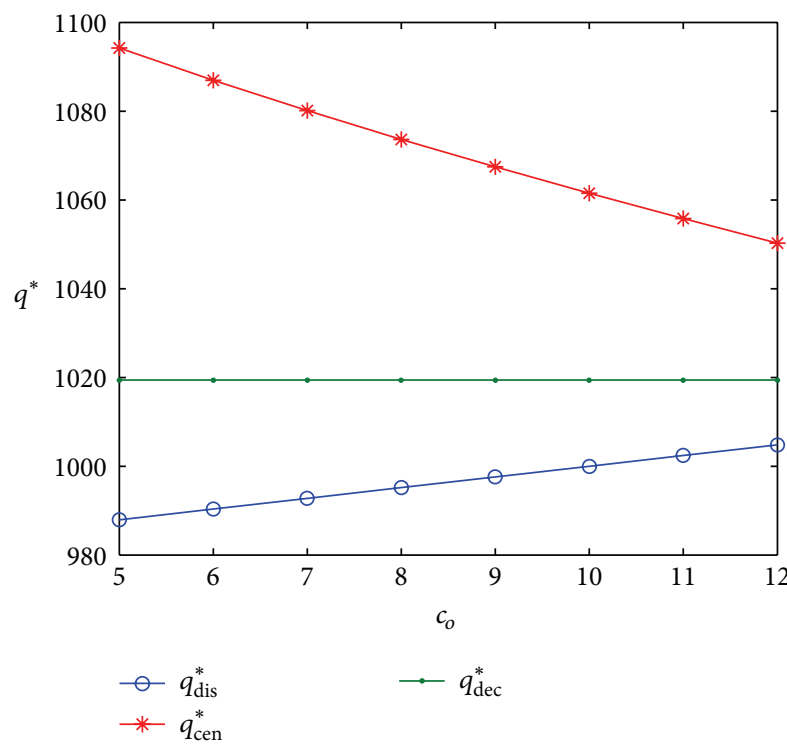

(a) The reservation quantities versus $c_{o}$

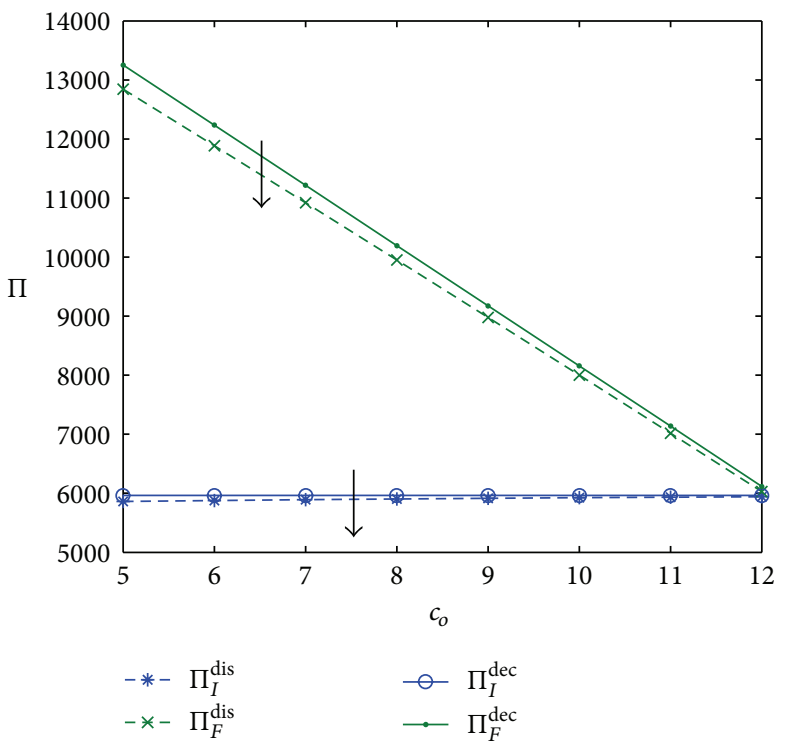

(b) The channel members' profits versus $c_{o}$

FIGURE 4: Impacts of $c_{o}$ on the reservation quantities and the channel members' profits under disadvantageous inequality.

seek a more fair trade even if his own profit will be hurt.

\subsection{Relevant Numerical Examples under Advantageous} Inequality. In this subsection, we give two numerical examples under advantageous inequality. One is to observe the change of the reservation quantities and the entire channel profit with respect to $\beta$ and $\gamma$. The other is to explore the impacts of the procurement cost, the operation cost, and the shortage cost on the reservation quantities and the channel members' profits.
Example 3. In this example, to satisfy $c_{s}>w>c_{o}>c_{m}$ and ensure that the profit of the LSI is larger than his equitable profit, we specify the parameters as follows: $c_{m}=4, c_{o}=14$, $w=16, c_{s}=22$, and $p=30$.

Figure 5 shows the results of the impacts of $\beta$ and $\gamma$ on the reservation quantities and the entire channel profits under advantageous inequality. Figure 5(a) shows that a generous LSI's reservation quantity will increase as $\beta$ or $\gamma$ increases, and when $\beta=1 /(1+\gamma)$ (e.g., $\beta=0.5, \gamma=1)$, the LSI's reservation quantity is equal to the centralized reservation 


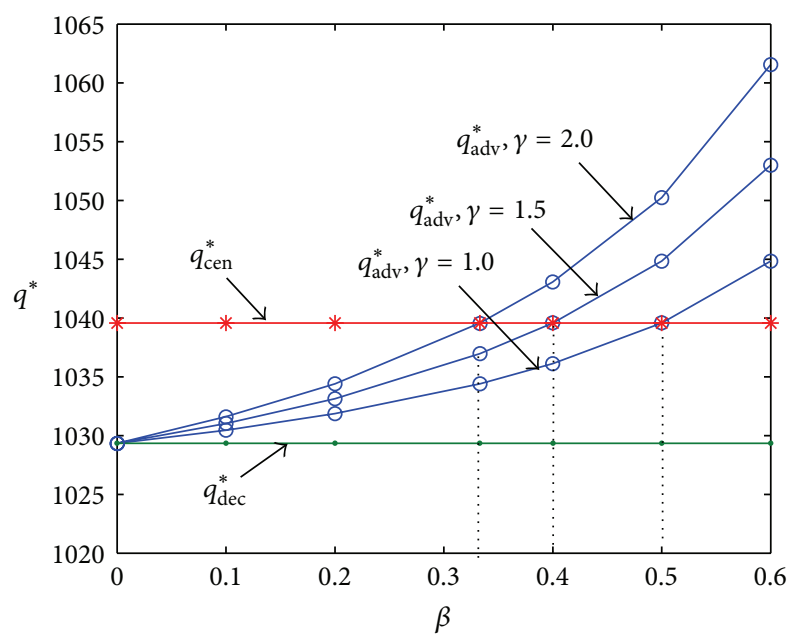

(a) The reservation quantities versus $(\beta, \gamma)$

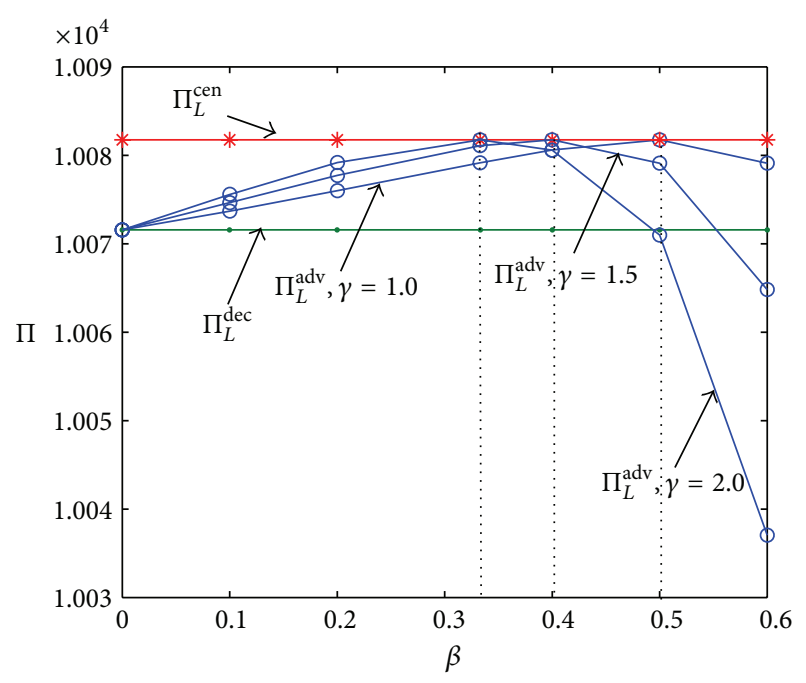

(b) The entire channel profits versus $(\beta, \gamma)$

FIGURE 5: Impacts of $\beta$ and $\gamma$ on the reservation quantities and the entire channel profits under advantageous inequality.

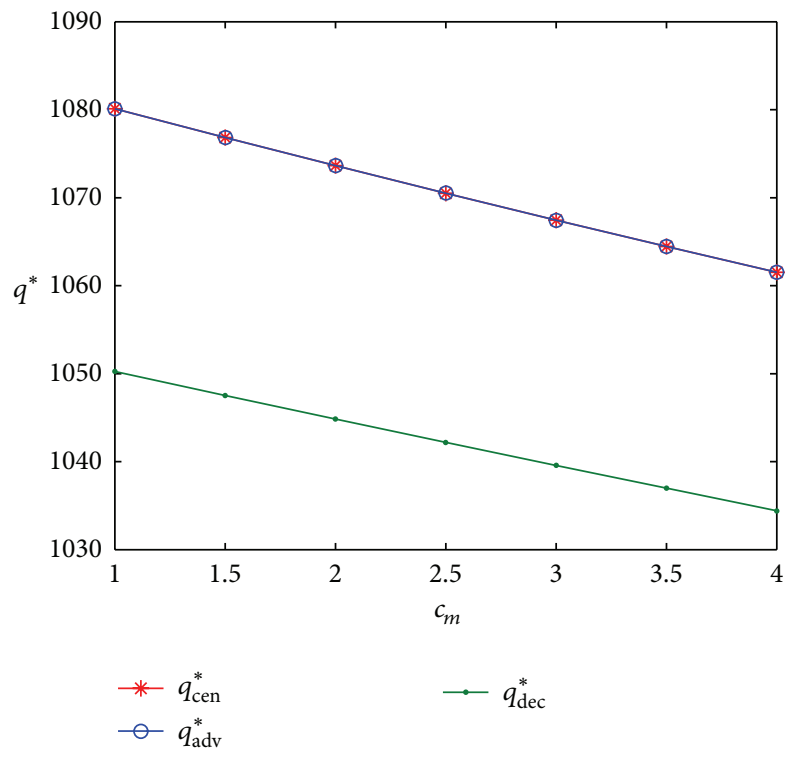

(a) The reservation quantity versus $c_{m}$

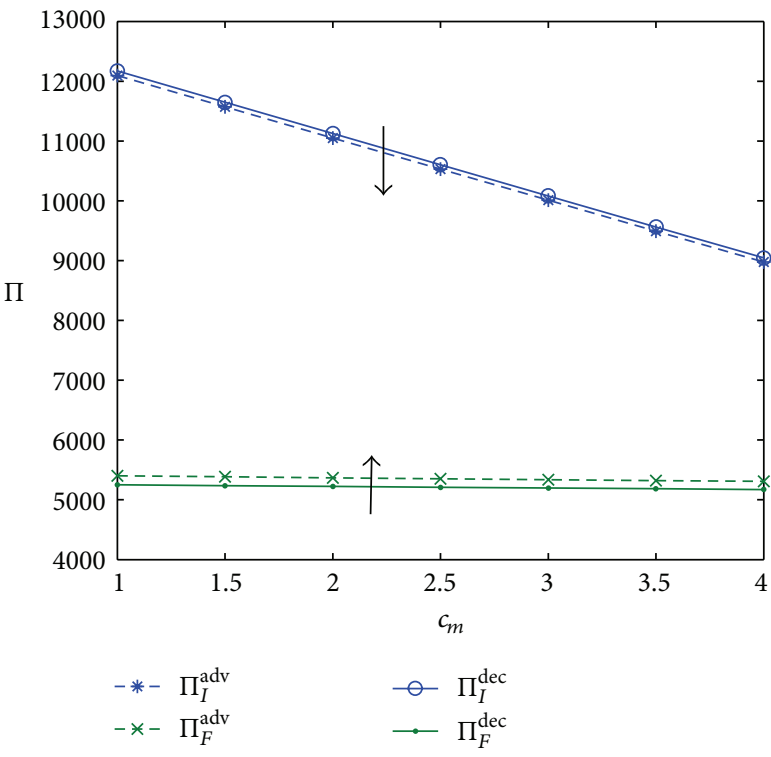

(b) The channel members' profits versus $c_{m}$

FIGURE 6: Impacts of $c_{m}$ on the reservation quantities and the channel members' profits under advantageous inequality.

quantity. The results are in accordance with Propositions 7 and 8. Figure 5(b) shows that the entire channel profit first increases and then decreases, with the increase of $\beta$. Also, there is the same conclusion for $\gamma$. When $\beta=1 /(1-\gamma)$ (e.g., $\beta=0.4, \gamma=1.5$ ), the entire channel profit under advantageous inequality is equal to the entire channel profit in the centralized setting; that is, the channel coordination in the LSSC can be achieved. Obviously, the results are in accordance with Propositions 9 and 11.

Example 4. In this example, we set three cases to investigate the impacts of $c_{m}, c_{s}$, and $c_{o}$ on the reservation quantities and channel members' profits under advantageous inequality. To satisfy $c_{s}>w>c_{o}>c_{m}$ and ensure that the profit of the LSI is larger than his equitable profit, we set the parameters for the three cases as follows.

Case 1. One has $c_{m} \in[1,4], \beta=0.5, \gamma=1, c_{o}=10, w=15$, $c_{s}=22$, and $p=30$.

Case 2. One has $c_{s} \in[18,25], \beta=0.5, \gamma=1, c_{m}=4, c_{o}=10$, $w=15$, and $p=30$.

Case 3. One has $c_{o} \in[8,14], \beta=0.5, \gamma=1, c_{m}=4, w=15$, $c_{s}=22$, and $p=30$.

For Case 1, the results are shown in Figure 6. From Figure 6(a), we can find that the reservation quantity of a generous LSI will decrease as the procurement cost increases. 


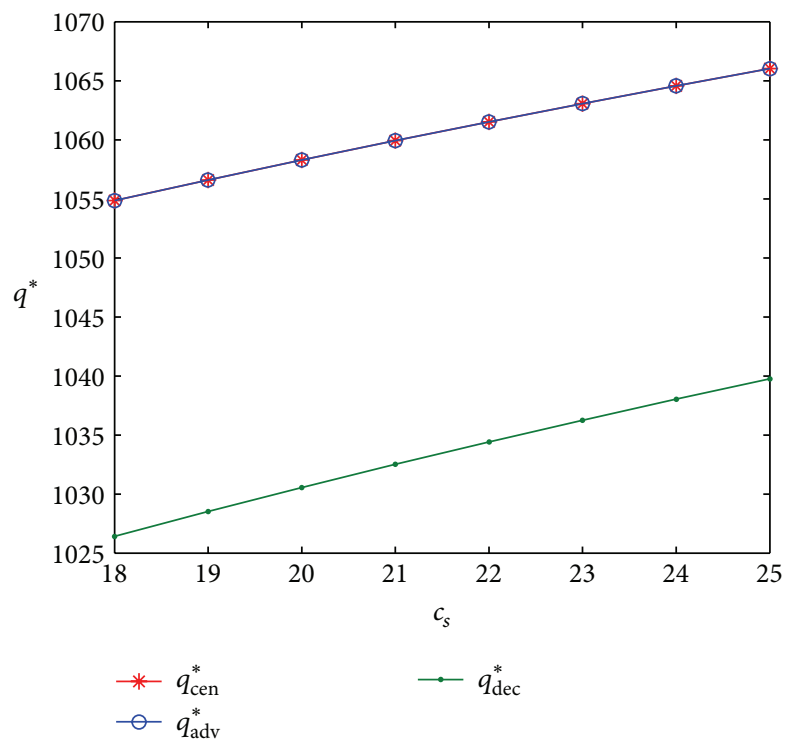

(a) The reservation quantities versus $c_{s}$

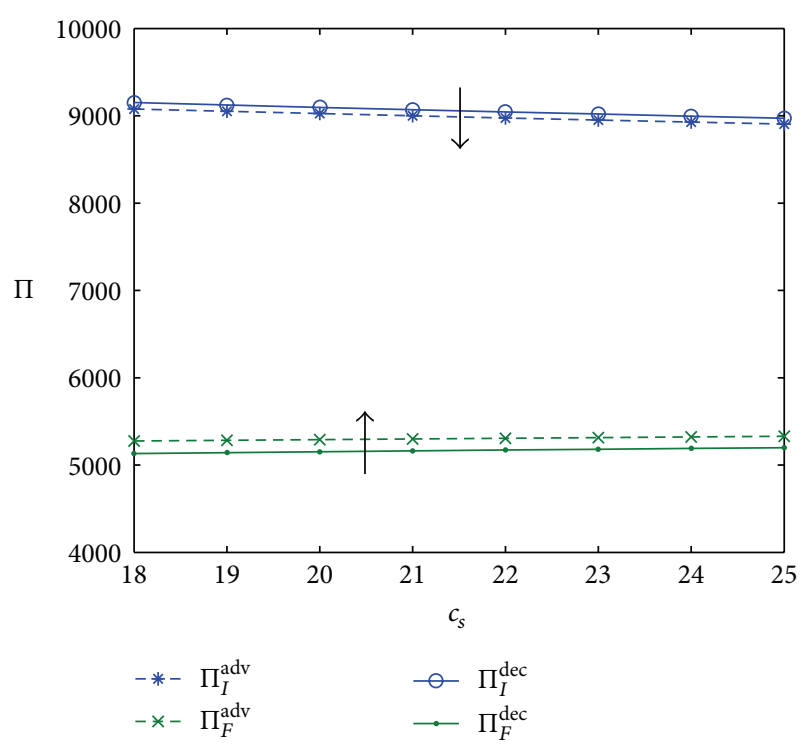

(b) The channel members' profits versus $c_{s}$

FIGURE 7: Impacts of $c_{s}$ on the reservation quantities and the channel members' profits under advantageous inequality.

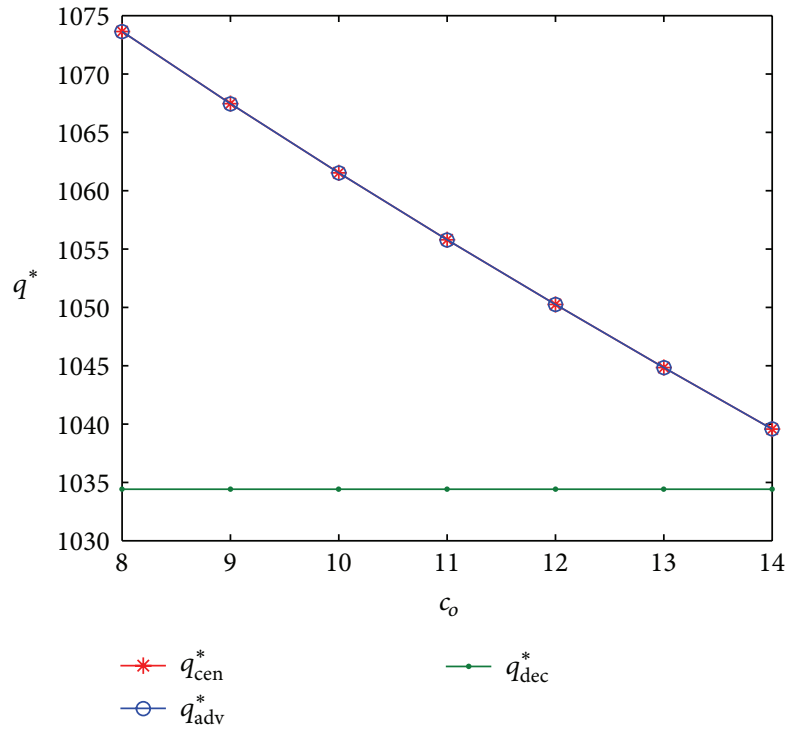

(a) The reservation quantities versus $c_{o}$

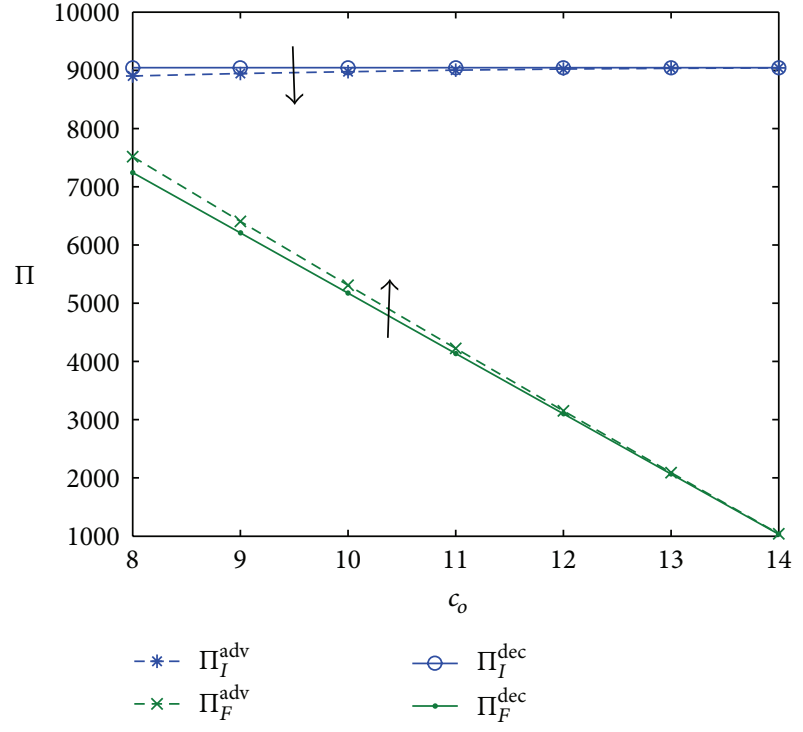

(b) The channel members' profits versus $c_{o}$

FIGURE 8: Impacts of $c_{o}$ on the reservation quantities and the channel members' profits under advantageous inequality.

Apparently, the results are in accordance with Proposition 9. Figure 6(b) shows that when the LSI experiences advantageous inequality, the profit of the LSI and the FLSP will decrease with the increase of the procurement cost.

For Case 2, the results are illustrated in Figure 7. Figure 7(a) shows that the reservation quantity of a generous LSI will increase as the shortage cost increases, and this result is in accordance with Proposition 9. Figure 7(b) shows that when the LSI experiences advantageous inequality, his profit will decrease with the increase of the shortage cost, while the FLSP's profit will increase.
For Case 3, the results are presented in Figure 8. Figure 8(a) shows that the reservation quantity of a generous LSI is affected by the FLSP's operation cost, and it is decreasing in $c_{o}$. The results are in accordance with Proposition 9. Figure 8(b) demonstrates that when the LSI experiences advantageous inequality, the LSI's profit will increase as the operation cost increases, while the FLSP's profit will decrease.

In addition, we can conclude from Figures 6(a), 7(a), and 8 (a) that the reservation quantity in the advantageous inequality scenario is equal to the centralized reservation 
quantity in the situation of $\beta=1 /(1+\gamma)$. This indicates that the coordination condition is not affected by the procurement cost, the shortage cost, or the operation cost. Also, it can be seen from Figures 6(b), 7(b), and 8(b) that the LSI's profit in the fair scenario is always smaller than that in the fair-neutral scenario, while for the FLSP, the conclusion is opposite. This indicates that if the LSI experiences advantageous inequality, he tends to reward the FLSP at the cost of decreasing his own profit so as to seek a more fair trade.

\section{Conclusions}

In this paper, fairness concerns are introduced into the analysis of channel coordination problem in a two-stage LSSC. Given the LSI's procurement cost and shortage cost, the FLSP's operation cost, and stochastic market demand, the models for a reservation price-only contract are established under disadvantageous inequality and advantageous inequality. The impacts of fairness concerns and the related costs on channel performance (i.e., the LSI's reservation quantity and channel profit) and channel coordination are analyzed. Compared with the existing literatures, the novelties of the paper are summarized in two aspects: one is that we capture the fairness factor in the collaborative cooperation between the logistics enterprises so as to get some new managerial insights to guide the practice; the other one is that we consider a more comprehensive situation when carrying out our fairness research; that is, the procurement cost, the potential shortage cost, and the salvage value are considered under stochastic market demand so as to lead the managers to take better decisions.

We find that channel coordination cannot be achieved by a simple reservation price-only contract in a fair-neutral scenario (i.e., both the LSI and the FLSP are fair-neutral) as well as in the situation that the LSI suffers from disadvantageous inequality, while channel coordination can be achieved under certain condition (see Proposition 11) if the LSI experiences advantageous inequality. This means that the double marginalization problem can be alleviated by the LSI's generosity. Our study also shows that, in the decentralized setting, the LSI's spite for disadvantageous inequality will result in less reservation quantity, while the LSI's generosity for advantageous inequality will result in greater reservation quantity. Meanwhile, the LSI's perceived relative advantage against the FLSP also plays an important role in alleviating (aggravating) the double marginalization problem and improving (worsening) the channel performance. Furthermore, another interesting finding shows that the LSI's reservation quantity is affected not only by his procurement cost and shortage cost but also by the FLSP's operation cost when the LSI perceives either disadvantageous or advantageous inequality. This just reflects that the LSI cares not only about his own profit but also about the FLSP's profit.

However, our study has some limitations, which may serve as directions for future research. First, in our study, the channel can be coordinated by the simple reservation priceonly contract under certain condition, but the condition rarely exists in reality. Hence, given fairness concerns, it seems interesting to find out some desirable contracts from multiple different contracts such as the buyback contract and the revenue-sharing contract. Second, we assume that the channel members have full information, which seems a strong assumption in reality [27]. Under the situation of information asymmetry, the channel coordination problem in the LSSC is a noteworthy research work. Last, we just study the channel coordination problem under the simple channel structure, that is, the LSSC composed of one LSI and one FLSP. It is necessary to further investigate the channel coordination problem under other complicated channel structures such as one LSI and two FLSPs.

\section{Appendix}

Proof of Proposition 1. By (10), we can obtain

$$
\begin{aligned}
\frac{\partial U_{\mathrm{dis}}^{I}}{\partial q} & =(1+\alpha) \Delta-\alpha \gamma\left(w-c_{o}\right), \\
\frac{\partial^{2} U_{\mathrm{dis}}^{I}}{\partial q^{2}} & =-(1+\alpha)\left(p+c_{s}\right) f(q),
\end{aligned}
$$

where $\Delta=\left(p+c_{s}\right)[1-F(q)]-\left(w+c_{m}\right)$. Because $f(q)>0$, we can get $\partial^{2} U_{\text {dis }}^{I} / \partial q^{2}<0$, so $U_{\text {dis }}^{I}$ is a strictly concave function of $q$. Let $\partial U_{\text {dis }}^{I} / \partial q=0$; we can obtain the optimal reservation quantity determined by (11).

Proof of Proposition 2. (a) By (11), we can get

$$
\frac{\partial q_{\mathrm{dis}}^{*}}{\partial \alpha}=\frac{d F^{-1}}{d E} \frac{\partial E}{\partial \alpha}=-\frac{d F^{-1}}{d E} \frac{\gamma\left(w-c_{o}\right)}{(1+\alpha)^{2}\left(p+c_{s}\right)},
$$

where

$$
E=1-\frac{(1+\alpha)\left(w+c_{m}\right)+\alpha \gamma\left(w-c_{o}\right)}{(1+\alpha)\left(p+c_{s}\right)}
$$

Since $F(x)$ and $F^{-1}(x)$ are monotonically increasing and $w>$ $c_{o}$, we have $\partial q_{\text {dis }}^{*} / \partial \alpha<0$; that is, $q_{\text {dis }}^{*}$ is decreasing in $\alpha$.

(b) Similarly, by (11), we can get

$$
\frac{\partial q_{\mathrm{dis}}^{*}}{\partial \gamma}=\frac{d F^{-1}}{d E} \frac{\partial E}{\partial \gamma}=-\frac{d F^{-1}}{d E} \frac{\alpha\left(w-c_{o}\right)}{(1+\alpha)\left(p+c_{s}\right)}<0 ;
$$

that is, $q_{\mathrm{dis}}^{*}$ is decreasing in $\gamma$.

(c) Since $\alpha>0, \gamma>0$, and $w>c_{o}$, it is known from (8) and (11) that

$$
1-\frac{w+c_{m}}{p+c_{s}}>1-\frac{(1+\alpha)\left(w+c_{m}\right)+\alpha \gamma\left(w-c_{o}\right)}{(1+\alpha)\left(p+c_{s}\right)}
$$

Since $F^{-1}(x)$ is monotonically increasing, we can get $q_{\text {dis }}^{*}<$ $q_{\mathrm{dec}}^{*}$. Above all, we have $q_{\mathrm{dis}}^{*}<q_{\mathrm{dec}}^{*}<q_{\mathrm{cen}}^{*}$. Thus, the proposition is proved. 
Proof of Proposition 3. By (11), we can get

$$
\begin{aligned}
\frac{\partial q_{\mathrm{dis}}^{*}}{\partial c_{m}} & =\frac{d F^{-1}}{d E} \frac{\partial E}{\partial c_{m}}=-\frac{d F^{-1}}{d E} \frac{1}{p+c_{s}}<0, \\
\frac{\partial q_{\mathrm{dis}}^{*}}{\partial c_{s}} & =\frac{d F^{-1}}{d E} \frac{\partial E}{\partial c_{s}} \\
& =\frac{d F^{-1}}{d E} \frac{(1+\alpha)\left(w+c_{m}\right)+\alpha \gamma\left(w-c_{o}\right)}{(1+\alpha)\left(p+c_{s}\right)^{2}}>0, \\
\frac{\partial q_{\mathrm{dis}}^{*}}{\partial c_{o}} & =\frac{d F^{-1}}{d E} \frac{\partial E}{\partial c_{o}}=\frac{d F^{-1}}{d E} \frac{\alpha \gamma}{(1+\alpha)\left(p+c_{s}\right)}>0,
\end{aligned}
$$

where

$$
E=1-\frac{(1+\alpha)\left(w+c_{m}\right)+\alpha \gamma\left(w-c_{o}\right)}{(1+\alpha)\left(p+c_{s}\right)} .
$$

It is concluded by the above formulas that Proposition 3 holds.

Proof of Proposition 4. Substituting $q_{\mathrm{dis}}^{*}$ determined by (11) into (3), (6), and (7) and taking the first derivative with respect to $\alpha$ and $\gamma$, respectively, we can get

$$
\begin{aligned}
& \frac{\partial \Pi_{I}^{\mathrm{dis}}}{\partial \alpha}=\varepsilon \frac{\partial q_{\mathrm{dis}}^{*}}{\partial \alpha}, \\
& \frac{\partial \Pi_{F}^{\mathrm{dis}}}{\partial \alpha}=\phi \frac{\partial q_{\mathrm{dis}}^{*}}{\partial \alpha}, \\
& \frac{\partial \Pi_{L}^{\mathrm{dis}}}{\partial \alpha}=\kappa \frac{\partial q_{\mathrm{dis}}^{*}}{\partial \alpha}, \\
& \frac{\partial \Pi_{I}^{\mathrm{dis}}}{\partial \gamma}=\varepsilon \frac{\partial q_{\mathrm{dis}}^{*}}{\partial \gamma} \\
& \frac{\partial \Pi_{F}^{\mathrm{dis}}}{\partial \gamma}=\phi \frac{\partial q_{\mathrm{dis}}^{*}}{\partial \gamma}, \\
& \frac{\partial \Pi_{L}^{\mathrm{dis}}}{\partial \gamma}=\kappa \frac{\partial q_{\mathrm{dis}}^{*}}{\partial \gamma},
\end{aligned}
$$

where $\varepsilon=\left(p+c_{s}\right)\left[1-F\left(q_{\text {dis }}^{*}\right)\right]-\left(w+c_{m}\right), \phi=w-c_{o}$, and $\kappa=\left(p+c_{s}\right)\left[1-F\left(q_{\text {dis }}^{*}\right)\right]-\left(c_{m}+c_{o}\right)$. According to Proposition 3, we know that $q_{\mathrm{dis}}^{*}<q_{\mathrm{dec}}^{*}<q_{\mathrm{cen}}^{*}$. By $q_{\mathrm{dis}}^{*}<q_{\mathrm{dec}}^{*}$ and (8) and (11), we can get $1-F\left(q_{\text {dis }}^{*}\right)>\left(w+c_{m}\right) /\left(p+c_{s}\right)$. By the above expression of $\varepsilon$, we can get $\varepsilon>0$. Since $w>c_{o}$, we know that $\phi>0$. By $q_{\text {dis }}^{*}<q_{\text {cen }}^{*}$ and (4) and (11), we can get $1-F\left(q_{\text {dis }}^{*}\right)>$ $\left(c_{m}+c_{o}\right) /\left(p+c_{s}\right)$. By the above expression of $\kappa$, we can get $\kappa>0$. According to the proof process of Proposition 2, we know that $\partial q_{\text {dis }}^{*} / \partial \alpha<0$; thus we can get $\partial \Pi_{I}^{\text {dis }} / \partial \alpha<0, \partial \Pi_{F}^{\text {dis }} / \partial \alpha<0$, and $\partial \Pi_{L}^{\text {dis }} / \partial \alpha<0$ if $\varepsilon>0, \phi>0$, and $\kappa>0$. Similarly, we can also obtain $\partial \Pi_{I}^{\text {dis }} / \partial \gamma<0, \partial \Pi_{F}^{\text {dis }} / \partial \gamma<0$, and $\partial \Pi_{L}^{\text {dis }} / \partial \gamma<0$. Hence, Proposition 4 holds.

Proof of Proposition 5. (a) By Section 3.1, we know that $\Pi_{I}$ is concave with respect to $q$ and $q_{\mathrm{dec}}^{*}$ is the optimal solution, and
$\Pi_{F}$ is increasing in $q$. By Proposition 2, we know that $q_{\text {dis }}^{*}<$ $q_{\mathrm{dec}}^{*}$. Substituting $q_{\mathrm{dis}}^{*}$ and $q_{\mathrm{dec}}^{*}$ into (6) and (7), respectively, we can get $\Pi_{I}^{\text {dis }}<\Pi_{I}^{\text {dec }}$ and $\Pi_{F}^{\text {dis }}<\Pi_{F}^{\text {dec }}$, respectively. Since $\Pi_{L}^{\mathrm{dis}}=\Pi_{I}^{\mathrm{dis}}+\Pi_{F}^{\mathrm{dis}}$ and $\Pi_{L}^{\mathrm{dec}}=\Pi_{I}^{\mathrm{dec}}+\Pi_{F}^{\mathrm{dec}}$, we can get $\Pi_{L}^{\mathrm{dis}}<$ $\Pi_{L}^{\mathrm{dec}}$.

(b) By Section 3.1, we know that $\Pi_{L}$ is concave with respect to $q$ and $q_{\text {cen }}^{*}$ is the optimal solution. By Proposition 3, we know that $q_{\text {dis }}^{*}<q_{\text {cen }}^{*}$. Substituting $q_{\text {dis }}^{*}$ and $q_{\text {cen }}^{*}$ into (3), respectively, we can get the corresponding $\Pi_{L}^{\text {dis }}$ and $\Pi_{L}^{\text {cen }}$. Thus, we can get $\Pi_{L}^{\text {dis }}<\Pi_{L}^{\text {cen }}$ because $q_{\text {dis }}^{*}<q_{\text {cen }}^{*}$. This means that channel coordination of the LSSC cannot be achieved. In other words, when channel coordination in the LSSC is realized, $w=c_{o}$ can be obtained by solving the equation $q_{\text {dis }}^{*}=q_{\text {cen }}^{*}$. In this case, the FLSP's profit will be zero. Yet this is inconsistent with the reality. Therefore, Proposition 5 holds.

Proof of Proposition 6. By (12), we can get

$$
\begin{gathered}
\frac{\partial U_{\mathrm{adv}}^{I}}{\partial q}=(1-\beta) \Delta+\beta \gamma\left(w-c_{o}\right), \\
\frac{\partial^{2} U_{\mathrm{adv}}^{I}}{\partial q^{2}}=-(1-\beta)\left(p+c_{s}\right) f(q),
\end{gathered}
$$

where $\Delta=\left(p+c_{s}\right)[1-F(q)]-\left(w+c_{m}\right)$. Since $f(q)>0$, we know that $\partial^{2} U_{\text {adv }}^{I} / \partial q^{2}<0$. So $U_{\text {adv }}^{I}$ is a strictly concave function of $q$. Letting $\partial U_{\text {adv }}^{I} / \partial q=0$, we can obtain the optimal reservation quantity determined by (13).

Proof of Proposition 7. (a) By (12), we can get

$$
\frac{\partial q_{\mathrm{adv}}^{*}}{\partial \beta}=\frac{d F^{-1}}{d H} \frac{\partial H}{\partial \beta}=\frac{d F^{-1}}{d H} \frac{\gamma\left(w-c_{o}\right)}{(1-\beta)^{2}\left(p+c_{s}\right)},
$$

where

$$
H=1-\frac{(1-\beta)\left(w+c_{m}\right)-\beta \gamma\left(w-c_{o}\right)}{(1-\beta)\left(p+c_{s}\right)} .
$$

Since $F(x)$ and $F^{-1}(x)$ are monotonically increasing and $w>$ $c_{o}$, we have $\partial q_{\mathrm{adv}}^{*} / \partial \beta>0$; that is, $q_{\mathrm{adv}}^{*}$ is increasing in $\beta$.

(b) By (8) and (12), we know that $q_{\mathrm{adv}}^{*}=q_{\mathrm{dec}}^{*}$ if $\beta=0$. Since $q_{\mathrm{adv}}^{*}$ is increasing in $\beta$, we know that $q_{\mathrm{dec}}^{*}<q_{\mathrm{adv}}^{*}$ if $0<$ $\beta<1$. Further, by (4) and (12), we know that $q_{\mathrm{adv}}^{*}=q_{\mathrm{cen}}^{*}$ if $\beta=1 /(1+\gamma)$. Thus, $q_{\mathrm{adv}}^{*}<q_{\mathrm{cen}}^{*}$ if $0<\beta<1 /(1+\gamma)$. Above all, we can get $q_{\mathrm{dec}}^{*}<q_{\mathrm{adv}}^{*}<q_{\mathrm{cen}}^{*}$ if $0<\beta<1 /(1+\gamma)$.

(c) According to the proof process of (b), we know that $q_{\mathrm{dec}}^{*}<q_{\mathrm{adv}}^{*}=q_{\mathrm{cen}}^{*}$ if $\beta=1 /(1+\gamma)$.

(d) Similarly, we know that $q_{\mathrm{adv}}^{*}>q_{\mathrm{cen}}^{*}>q_{\mathrm{dec}}^{*}$ if $1 /(1+\gamma)<$ $\beta<1$. Therefore, the proposition is proved.

Proof of Proposition 8. The proof of Proposition 8 is similar to that of Proposition 7 and thus omitted. 
Proof of Proposition 9. By (13), we can get

$$
\begin{aligned}
& \frac{\partial q_{\mathrm{adv}}^{*}}{\partial c_{m}}=\frac{d F^{-1}}{d H} \frac{\partial H}{\partial c_{m}}=-\frac{d F^{-1}}{d H} \frac{1}{p+c_{s}}<0, \\
& \frac{\partial q_{\mathrm{adv}}^{*}}{\partial c_{s}}=-\frac{\partial^{2} U_{I} / \partial q \partial c_{s}}{\partial^{2} U_{I} / \partial q^{2}}=\frac{1-F(q)}{\left(p+c_{s}\right) f(q)}>0, \\
& \frac{\partial q_{\mathrm{adv}}^{*}}{\partial c_{o}}=\frac{d F^{-1}}{d H} \frac{\partial H}{\partial c_{o}}=-\frac{d F^{-1}}{d H} \frac{\beta \gamma}{(1-\beta)\left(p+c_{s}\right)}<0,
\end{aligned}
$$

where

$$
H=1-\frac{(1-\beta)\left(w+c_{m}\right)-\beta \gamma\left(w-c_{o}\right)}{(1-\beta)\left(p+c_{s}\right)} .
$$

Hence, Proposition 9 holds.

Proof of Proposition 10. We substitute $q_{\mathrm{adv}}^{*}$ determined by (13) into (3), (6), and (7) and take the first derivative of $\Pi_{L}^{\mathrm{adv}}, \Pi_{I}^{\mathrm{adv}}$, and $\Pi_{F}^{\mathrm{adv}}$ with respect to $\beta$ and $\gamma$, respectively. Thus, we can get

$$
\begin{aligned}
& \frac{\partial \Pi_{L}^{\mathrm{adv}}}{\partial \beta}=\kappa \frac{\partial q_{\mathrm{adv}}^{*}}{\partial \beta}, \\
& \frac{\partial \Pi_{I}^{\mathrm{adv}}}{\partial \beta}=\varepsilon \frac{\partial q_{\mathrm{adv}}^{*}}{\partial \beta}, \\
& \frac{\partial \Pi_{F}^{\mathrm{adv}}}{\partial \beta}=\phi \frac{\partial q_{\mathrm{adv}}^{*}}{\partial \beta}, \\
& \frac{\partial \Pi_{L}^{\mathrm{adv}}}{\partial \gamma}=\kappa \frac{\partial q_{\mathrm{adv}}^{*}}{\partial \gamma}, \\
& \frac{\partial \Pi_{I}^{\mathrm{adv}}}{\partial \gamma}=\varepsilon \frac{\partial q_{\mathrm{adv}}^{*}}{\partial \gamma}, \\
& \frac{\partial \Pi_{F}^{\mathrm{adv}}}{\partial \gamma}=\phi \frac{\partial q_{\mathrm{adv}}^{*}}{\partial \gamma},
\end{aligned}
$$

where $\varepsilon=\left(p+c_{s}\right)\left[1-F\left(q_{\mathrm{adv}}^{*}\right)\right]-\left(w+c_{m}\right), \phi=w-c_{o}>0$, and $\kappa=\left(p+c_{s}\right)\left[1-F\left(q_{\mathrm{adv}}^{*}\right)\right]-\left(c_{m}+c_{o}\right)$. By Proposition 7 , we know that $q_{\mathrm{adv}}^{*}>q_{\mathrm{dec}}^{*}$. Thus, according to (8) and (13), we can get $1-F\left(q_{\mathrm{adv}}^{*}\right)<\left(w+c_{m}\right) /\left(p+c_{s}\right)$. According to this inequation, we can get $\varepsilon<0$. By Proposition 7 , we know that $q_{\text {adv }}^{*}<q_{\text {cen }}^{*}$ if $0<\beta<1 /(1+\gamma)$ and $q_{\mathrm{adv}}^{*}>q_{\mathrm{cen}}^{*}$ if $1 /(1+\gamma)<\beta<1$. Thus, from (4) and (13), we can get $1-F\left(q_{\mathrm{adv}}^{*}\right)>\left(c_{m}+c_{o}\right) /\left(p+c_{s}\right)$ if $0<\beta<1 /(1+\gamma)$ and $1-F\left(q_{\mathrm{adv}}^{*}\right)<\left(c_{m}+c_{o}\right) /\left(p+c_{s}\right)$ if $1 /(1+\gamma)<\beta<1$. According to these two inequations, we can get $\kappa>0(\kappa<0)$ if $0<\beta<1 /(1+\gamma)(1 /(1+\gamma)<\beta<1)$. By the proof process of Proposition 7, we know that $\partial q_{\mathrm{adv}}^{*} / \partial \beta>0$. Thus, we can, respectively, get $\partial \Pi_{I}^{\mathrm{adv}} / \partial \beta<0, \partial \Pi_{F}^{\mathrm{adv}} / \partial \beta>0$, and $\partial \Pi_{L}^{\text {adv }} / \partial \beta>0$ if $0<\beta<1 /(1+\gamma)$ and $\partial \Pi_{L}^{\text {adv }} / \partial \beta<0$ if $1 /(1+\gamma)<\beta<1$. Similarly, we also can, respectively, get $\partial \Pi_{I}^{\mathrm{adv}} / \partial \gamma<0, \partial \Pi_{F}^{\mathrm{adv}} / \partial \gamma>0$, and $\partial \Pi_{L}^{\mathrm{adv}} / \partial \gamma>0$ if $0<\gamma<$ $(1-\beta) / \beta$ and $\partial \Pi_{L}^{\text {adv }} / \partial \gamma<0$ if $\gamma>(1-\beta) / \beta$. This completes the proof of Proposition 10.
Proof of Proposition 11. By Section 3.1, we know that $\Pi_{L}$ is concave with respect to $q$. By Proposition 7 , we know that $q_{\mathrm{adv}}^{*}=q_{\mathrm{cen}}^{*}$ if $\beta=1 /(1+\gamma)$. Substituting $q_{\mathrm{cen}}^{*}$ determined by (4) and $q_{\mathrm{adv}}^{*}$ determined by (13) into (3), respectively, we can get the corresponding $\Pi_{L}^{\mathrm{cen}}$ and $\Pi_{L}^{\mathrm{adv}}$. Thus, if $q_{\mathrm{adv}}^{*}=q_{\mathrm{cen}}^{*}$, then we know $\Pi_{L}^{\mathrm{adv}}=\Pi_{L}^{\mathrm{cen}}$; that is, Proposition 11 holds.

\section{Competing Interests}

The authors declare that they have no competing interests.

\section{Acknowledgments}

This work was partly supported by the National Natural Science Foundation of China (Projects nos. 71271051, 71571039, and 71201020) and the Fundamental Research Funds for the Central Universities, NEU, China (Projects nos. N140607001, N140604003, and N150606001).

\section{References}

[1] W. H. Liu, C. L. Liu, X. C. Xu, and X. Bao, "An order allocation model in multi-period logistics service supply chain based on cumulative prospect theory and capacity matching constraint," International Journal of Production Research, vol. 52, no. 22, pp. 6608-6626, 2014.

[2] M. He, J. Xie, X. Wu, Q. Hu, and Y. Dai, "Capability coordination in automobile logistics service supply chain based on reliability," Procedia Engineering, vol. 137, pp. 325-333, 2016.

[3] K. L. Choy, C.-L. Li, S. C. K. So, H. Lau, S. K. Kwok, and D. W. K. Leung, "Managing uncertainty in logistics service supply chain," International Journal of Risk Assessment and Management, vol. 7, no. 1, pp. 19-43, 2007.

[4] W.-H. Liu, X.-C. Xu, and A. Kouhpaenejad, "Deterministic approach to the fairest revenue sharing coefficient in logistics service supply chain under the stochastic demand condition," Computers \& Industrial Engineering, vol. 66, no. 1, pp. 41-52, 2013.

[5] J. G. V. Vieira, J. C. Fransoo, and C. D. Carvalho, "Freight distribution in megacities: perspectives of shippers, logistics service providers and carriers," Journal of Transport Geography, vol. 46, pp. 46-54, 2015.

[6] B. M. Beamon, "Designing the green supply chain," Logistics Information Management, vol. 12, no. 4, pp. 332-342, 1999.

[7] R. H. Ballou, S. M. Gilbert, and A. Mukherjee, "New managerial challenges from supply chain opportunities," Industrial Marketing Management, vol. 29, no. 1, pp. 7-18, 2000.

[8] L. M. Ellram, W. L. Tate, and C. Billington, "Understanding and managing the services supply chain," Journal of Supply Chain Management, vol. 40, no. 4, pp. 17-32, 2004.

[9] J. S. Arlbjørn, P. V. Freytag, and H. de Haas, "Service supply Chain management: a survey of lean application in the municipal sector," International Journal of Physical Distribution \& Logistics Management, vol. 41, no. 3, pp. 277-295, 2011.

[10] M. Giannakis, "Management of service supply chains with a service-oriented reference model: the case of management consulting," Supply Chain Management, vol. 16, no. 5, pp. 346361, 2011.

[11] H. F. Zhao, B. Lin, W. Q. Mao, and Y. Ye, "Differential game analyses of logistics service supply chain coordination by cost 
sharing contract," Journal of Applied Mathematics, vol. 2014, Article ID 842409, 10 pages, 2014.

[12] G. P. Cachon, "Supply chain coordination with contracts," in Handbooks in Operations Research and Management Science, vol. 11, pp. 227-339, 2003.

[13] S. J. Sang, "Supply chain contracts with multiple retailers in a fuzzy demand environment," Mathematical Problems in Engineering, vol. 2013, Article ID 482353, 12 pages, 2013.

[14] S. S. Sana, "A collaborating inventory model in a supply chain," Economic Modelling, vol. 29, no. 5, pp. 2016-2023, 2012.

[15] T. H. Cui, J. S. Raju, and Z. J. Zhang, "Fairness and channel coordination," Management Science, vol. 53, no. 8, pp. 1303-1314, 2007.

[16] S. F. Du, T. F. Nie, C. B. Chu, and Y. Yu, "Newsvendor model for a dyadic supply chain with Nash bargaining fairness concerns," International Journal of Production Research, vol. 52, no. 17, pp. 5070-5085, 2014.

[17] T.-H. Ho, X. Su, and Y. Wu, "Distributional and peer-induced fairness in supply chain contract design," Production and Operations Management, vol. 23, no. 2, pp. 161-175, 2014.

[18] X. L. Wu and J. A. Niederhoff, "Fairness in selling to the newsvendor," Production and Operations Management, vol. 23, no. 11, pp. 2002-2022, 2014.

[19] N. Kumar, L. K. Scheer, and J. E. Steenkamp, "The effects of supplier fairness on vulnerable resellers," Journal of Marketing Research, vol. 32, no. 1, pp. 54-65, 1995.

[20] D. Corsten and N. Kumar, "Do suppliers benefit from collaborative relationships with large retailers? An empirical investigation of efficient consumer response adoption," Journal of Marketing, vol. 69, no. 3, pp. 80-94, 2005.

[21] C. H. Loch and Y. Wu, "Social preferences and supply chain performance: an experimental study," Management Science, vol. 54, no. 11, pp. 1835-1849, 2008.

[22] M. Rabin, "Incorporating fairness into game theory and economics," The American Economic Review, vol. 83, no. 5, pp. 12811302, 2011.

[23] L. K. Scheer, N. Kumar, and J.-B. E. M. Steenkamp, "Reactions to perceived inequity in U.S. and Dutch interorganizational relationships," Academy of Management Journal, vol. 46, no. 3, pp. 303-316, 2003.

[24] J. Yang, J. X. Xie, X. X. Deng, and H. Xiong, "Cooperative advertising in a distribution channel with fairness concerns," European Journal of Operational Research, vol. 227, no. 2, pp. 401-407, 2013.

[25] W.-H. Liu, D. Xie, and X.-C. Xu, "Quality supervision and coordination of logistic service supply chain under multi-period conditions," International Journal of Production Economics, vol. 142, no. 2, pp. 353-361, 2013.

[26] O. Caliskan-Demirag, Y. F. Chen, and J. Li, "Channel coordination under fairness concerns and nonlinear demand," European Journal of Operational Research, vol. 207, no. 3, pp. 1321-1326, 2010.

[27] E. Katok, T. Olsen, and V. Pavlov, "Wholesale pricing under mild and privately known concerns for fairness," Production and Operations Management, vol. 23, no. 2, pp. 285-302, 2014.

[28] B. Holmstrom and P. Milgrom, "Aggregation and linearity in the provision of intertemporal incentives," Econometrica, vol. 55, no. 2, pp. 303-328, 1987.

[29] B. Kalkanci, K.-Y. Chen, and F. Erhun, "Contract complexity and performance under asymmetric demand information: an experimental evaluation," Management Science, vol. 57, no. 4, pp. 689-704, 2011.
[30] B. Kalkanci, K.-Y. Chen, and F. Erhun, "Complexity as a contract design factor: a human-to-human experimental study," Production and Operations Management, vol. 23, no. 2, pp. 269284, 2014.

[31] C. L. Williams and B. E. Patuwo, "Analysis of the effect of various unit costs on the optimal incoming quantity in a perishable inventory model," European Journal of Operational Research, vol. 156, no. 1, pp. 140-147, 2004.

[32] S. S. Sana, "An EOQ model for stochastic demand for limited capacity of own warehouse," Annals of Operations Research, vol. 233, pp. 383-399, 2015.

[33] B. Pal, S. S. Sana, and K. Chaudhuri, "A distribution-free newsvendor problem with nonlinear holding cost," International Journal of Systems Science, vol. 46, no. 7, pp. 1269-1277, 2015.

[34] E. Fehr and K. M. Schmidt, "A theory of fairness, competition, and cooperation," Quarterly Journal of Economics, vol. 114, no. 3, pp. 817-868, 1999.

[35] D. Gao, X. Zhao, and W. Geng, "A delay-in-payment contract for Pareto improvement of a supply chain with stochastic demand," Omega, vol. 49, pp. 60-68, 2014. 


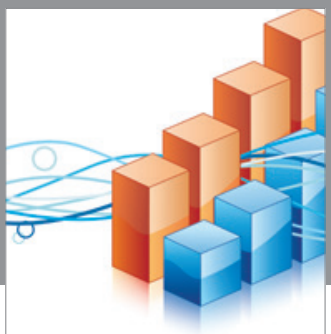

Advances in

Operations Research

vatem alat4

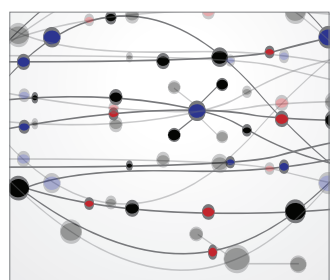

\section{The Scientific} World Journal
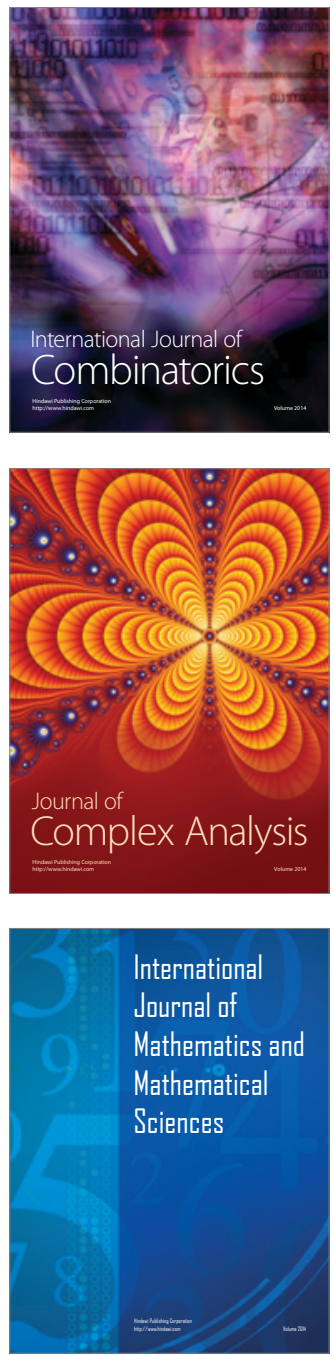
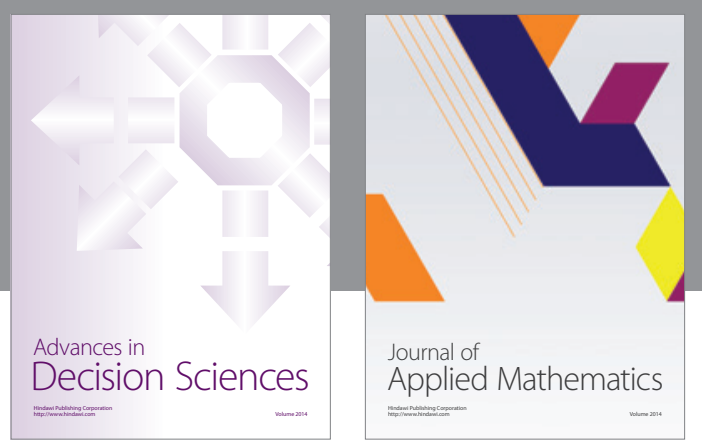

Algebra

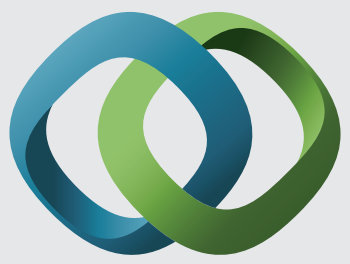

\section{Hindawi}

Submit your manuscripts at

http://www.hindawi.com
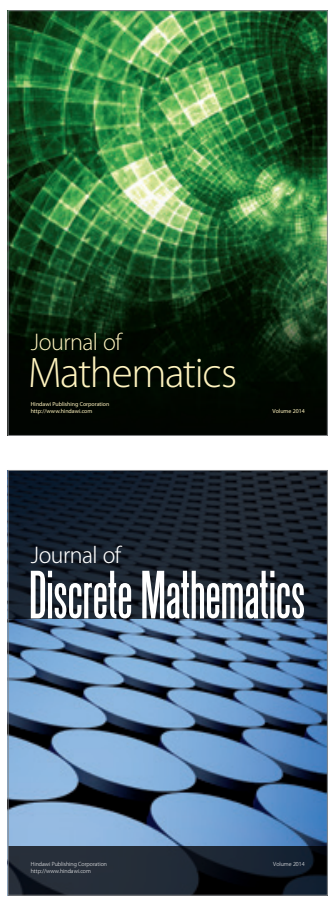

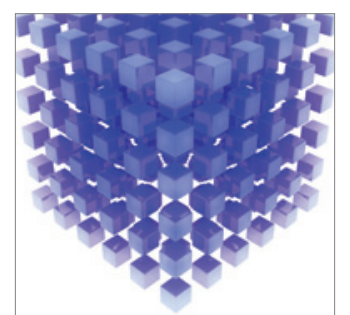

Mathematical Problems in Engineering
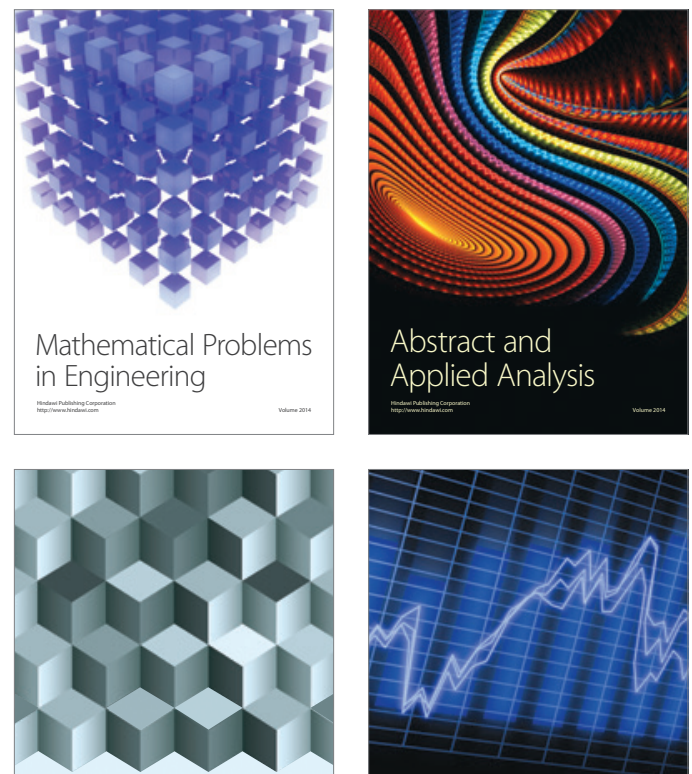

Journal of

Function Spaces

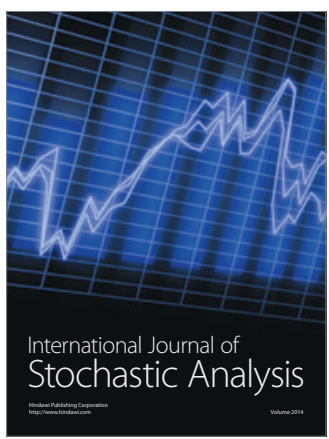

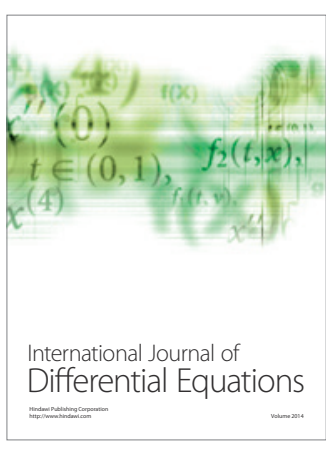
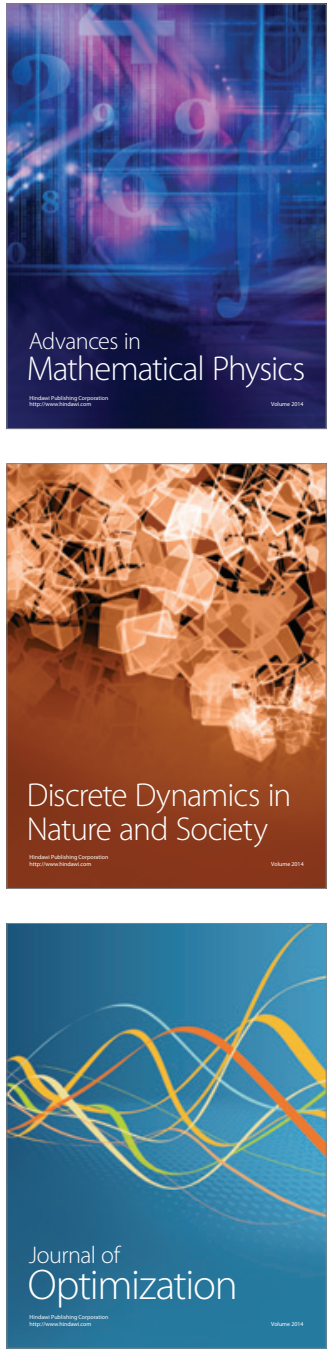